\title{
The Study of the Refractive Index in Anisotropic Absorptive Turbulent Magnetized Plasma
}

\author{
Ilir F. Progri \\ Giftet Inc., 5 Euclid Ave. \#3, Worcester, MA 01610, USA \\ ORCID: 0000-0001-5197-1278
}

Correspondence should be addressed to Ilir Progri; iprogri@giftet.com

Received December 8, 2017; Revised December 19, 21, 2017-January 7, 2018, April 9-26, 2019; Accepted July 16, 2019;

Published November 1, 2019.

Scientific Editor-in-Chief/Editor: Ilir F. Progri

Copyright (C) 2019 Giftet Inc. All rights reserved. This work may not be translated or copied in whole or in part without written permission to the publisher (Giftet Inc., 5 Euclid Ave. \#3, Worcester, MA 01610, USA), except for brief excerpts in connection with reviews or scholarly analysis. Use in connection with any form of information storage and retrieval, electronic adaptation, computer software or by similar or dissimilar methodology now known or hereafter developed is forbidden. The use of the publication of trade names, trademarks, service marks, or similar terms, even if they are not identified as such, is not to be taken as an expression of opinion as to whether or not they are subject to proprietary rights.

The study of the refractive index in anisotropic absorptive turbulent magnetized plasma is performed analytically and numerically for two important scenarios: without collision and with collision. This journal paper attempt to assess what Appleton had in mind when he stated that "The somewhat complicated [refractive index] formula (35) [1932, [2]] may be simplified for certain practical cases." The refractive index lays the foundation of the radio wave propagation in uniform magneto-ionic plasma. Therefore, it is attempted to perform an outstanding derivation, simplification, simulation, and classification of the Appleton-Hartree magneto-ionic formula not published anywhere else.

Index Terms - Refractive index, anisotropic, absorptive, turbulent, magnetized, plasma, collision, complex numbers, tensor analysis, time-invariant, time-variant.

\section{Introduction}

In 1925, Appleton and Barnett published their first major discovery that explains why: "(a) long distance wireless communications is possible, and (b) large and rapid variations of signal intensity and apparent direction of propagation of the waves are observed at night, and, to some extent, during daylight, particularly in winter" [1].

In 1932, Appleton published the iconic paper on "wireless studies of the ionosphere" where he provides evidence from both theory and experimental results on the existence of the ionosphere and the nature of the refractive index of the ionosphere [2].

Since Appleton iconic paper (1932, [2]), many scientists, researcher, and scholars have derived, re-derived, studied, computed, etc. the refractive index [3]-[30], [48], [49] either using the Maxwell equations, or through the solution of the Boltzmann equation following the Chapman-Enskog method 


\section{[2], [4]-[10], [14], [19].}

This paper discusses the study of the refractive index in anisotropic absorptive turbulent magnetized plasma performed analytically and numerically for two important scenarios: collision-less and with collision invariant of time.

There are various accounts [1]-[3], [8], [13], [15], [18]-[21], that describe the main achievements that led to the discovery of the ionosphere (a.k.a. cold and drifting plasma).

The main objective of this paper is twofold. First, a complete, thorough, and detailed review ${ }^{\mathrm{i}}$, investigation, examination, and assessment of Appleton-Hartree-Lassen magneto-iconic equation [1],[2],[19], in light of all the evidence [3]-[30] that serves as a verification of Jandieri et al, 2007 [14]. During this process the analysis of various important existing formulas and new formulas is performed.

Why is this important? It is well known that the refractive index changes as an electromagnetic wave propagates through ionosphere whose manner is investigated in this journal paper. In general this change is a random process; however, certain main assumptions are applied to simplify the theoretical model: stationary or time-invariant or stochastic or time-variant; collision-less or without collision or with collision.

Why are these assumptions important and how do they relate to refractive index in real-time? These assumptions are important because they serve as the foundation for establishing certain main scenarios that describe the physical condition of the cold plasma and therefore determine how electromagnetic wave propagation is affected by the plasma and ultimately the closed form expression of refractive index of the ionosphere. The time-invariant or stationary assumption corresponds to a quiet, storm-less, motionless, or noiseless plasma; i.e., a plasma condition not affected by noise or the physical motion of any outside disturbance such as a typically a sun-storm or cosmic storm. The time-variant assumption corresponding to a plasma condition affected by the outside disturbance such as a day, a sun-storm or a cosmic storm is not considered in this paper.

The assumption related to collision is more or less an artificial assumption as there is always some kind of collision going on in the plasma. If we were to derive the two scenarios one collision-less and one with collision we should come up with two different closed form expressions. Then if we were to take the expression with collision and assume that collision is zero we should be able to arrive at the collision-less closed form expression.

One of the main focuses of this paper serves as a means of verification to what Appleton stated: "The somewhat complicated [refractive index] formula (35) [1932, [2]] may be simplified for certain practical cases." Therefore, my main motivation with this paper is to understand the thoughts of Appleton and to be able to explain to the readers today the degree of the simplifications.

In spring of 1996, I took a graduate course in Electromagnetic Theory [9] as part of my Master Degree program at the Electrical and Computer Engineering Department, Worcester Polytechnic Institute, Worcester, MA. As part of my education which consisted of homework assignments, take home exams and research projects, I developed a profound understanding of the derivations of the Appleton-Hartree magneto-iconic formula and the approach performed by Prof. Kong, 1990, [9] so much so that Prof. Reinhold Ludwig was beyond excited with my performance in the class. To some respect, this study may also serve as an extension of the work published by Prof. Kong, 1990, [9].

In 2003 after completing my Ph.D. Dissertation in "An assessment of indoor geolocation systems" (Progri 2003, [12]), I became interested in extending (or expanding) the capability of the indoor signals to GPS satellite signals [55] which will hopefully get published in my pioneer publication soon [54]. Since 2013 I came into contact with a very interesting journal paper from Jandieri et al., (2007, [14]) which provides some understanding of the plasma amplitude and phase variances. I believed that a full and complete investigation of the Jandieri's publication was needed before I would attempt to understand and simulate his findings in MATLAB [56]. So, I did perform a thorough investigation of Jandieri et al., (2007, [14]) and I communicated my findings to Jandieri himself. I believe I told him why I was so interested in his work and also that there were some typos in his manuscript. He completely rejected my proposal but later on he accepted that indeed there were some typos in his published paper. Anyway, we agreed to work together on one of his projects but as soon as I started my journal and I offered him an editorial position in my journal he rejected the idea and we have not communicated since then. I believe his reason for rejection was that he did not want to expose his work to further criticism from me.

Anyway, this work is entirely original work that I have performed based on my earlier derivations dating back since 1996 and I believe that this publication falls into the category of masterpiece marvels in electromagnetic theory series.

Let us also clarify the other assumptions made about the 
plasma and also about the refractive index. Ionospheric plasma is assumed:

1. anisotropic; i.e., the opposite of isotropic; i.e., plasma physical properties vary with direction. Due to the influence of the anisotropic Earth' magnetic field, the ionosphere becomes anisotropic so that the ionospheric reflection factor in reality is a matrix; hence, this effect produces the permittivity of anisotropic media tensor [22].

2. absorptive; i.e., having power or capacity or tendency to absorb or soak up the electromagnetic wave energy. Ionospheric absorption (or ISAB) is the scientific name for absorption occurring as a result of the interaction between various types of electromagnetic waves and the free electrons in the ionosphere, which can interfere with radio transmissions [23]. We have modeled this effect by means of the extinction coefficient.

3. turbulent; i.e., characterized by disorder, or collision; not controlled or calm; i.e., not particle collision-less. We have modeled this effect my means of the electron collision frequency.

4. magnetized; i.e., affected by the earth magnetic field or an outside or external magnetic field or force. We have modeled this effect by means of a magnetic flux density vector. Others have used the magnetic intensity vector as in the case of (Jandieri et al., 2007, [14]), and (Karia, Pathak, 2010, [28]).

This paper is organized as follows: in Sect. 2 the refractive index in anisotropic absorptive collision-less calm magnetized plasma is discussed. The refractive index in anisotropic absorptive turbulent magnetized plasma is discussed in Sect. 3. Section 4 contains numerical, theoretical results; Conclusion is provided in Sect. 5 along with a list of references. In appendix A the derivations of the simplifications of refractive index given by (76) are performed. The derivations of Kong (14) pg. 78 is given in appendix B. In appendix $\mathrm{C}$ a brief discussion on electron collision frequency is performed. The explanation of differences between (100) and (102) is derived in appendix D. In appendix E, simplification calculations with complex numbers (108) through (110) are performed. In Appendix F, the substitution of $\kappa, \kappa \_g$, and $\kappa \_z$ given by (111) through (113) in (68) is provided. In Appendix $G$, the simplification of Appleton-Hartree magneto-ionic formula given by Progri (116) is performed. In Appendix H special cases are discussed. In appendix I: the derivation of the extinction coefficient in the absence of magnetic field is performed.

\section{Refractive Index in Anisotropic Absorptive Particle Calm Magnetized Plasma}

The simplest model to compute the refractive index is in anisotropic absorptive particle calm magnetized plasma ${ }^{\text {ii }}$ (ex. Jandieri's [14] used in (cgs ${ }^{\mathrm{iii}}$ units [31])).

Consider an electron plasma placed in a dc magnetic field $\mathbf{B}_{0}$. The electron is subject to a force $\mathbf{F}=e\left(\mathbf{E}+\mathbf{v} \times \mathbf{B}_{0}\right)$ under an external dc magnetic flux density vector $\mathbf{B}_{0}$. From Newton's second law $\mathbf{F}=\frac{d\left(m_{e} \mathbf{v}\right)}{d t}$. Under time-harmonic excitation and for small $\mathbf{v}$; we approximate the total time derivative by $-i \omega$ and arrive at the relations between $\mathbf{E}, \mathbf{v}$, and $\mathbf{B}_{0}$ as follows:

$-i \omega m_{e} \mathbf{v}=e\left(\mathbf{E}+\mathbf{v} \times \mathbf{B}_{0}\right)$

Let us apply the cross product to (1) so we obtain:

$-i \omega m_{e}\left(\mathbf{B}_{0} \times \mathbf{v}\right)=e\left[\mathbf{B}_{0} \times \mathbf{E}+\underline{\mathbf{B}_{0} \times\left(\mathbf{v} \times \mathbf{B}_{0}\right)}\right]$

Let us consider the underline vector, $\mathbf{B}_{0} \times\left(\mathbf{v} \times \mathbf{B}_{0}\right)$, as follows:

$\underline{\mathbf{B}_{0} \times\left(\mathbf{v} \times \mathbf{B}_{0}\right)}=\left(\mathbf{B}_{0} \cdot \mathbf{B}_{0}\right) \mathbf{v}-\left(\mathbf{B}_{0} \cdot \mathbf{v}\right) \mathbf{B}_{0}$

Hence, substituting (3) into (2) we obtain

$-i \omega m_{e}\left(\mathbf{B}_{0} \times \mathbf{v}\right)=e\left[\mathbf{B}_{0} \times \mathbf{E}+\left(\mathbf{B}_{0} \cdot \mathbf{B}_{0}\right) \mathbf{v}-\underline{\underline{\left(\mathbf{B}_{0} \cdot \mathbf{v}\right)}} \mathbf{B}_{0}\right]$

Next, in order to evaluate the second underline dot product, $\left(\mathbf{B}_{0} \cdot \mathbf{v}\right)$, we dot the first product (1) with $\mathbf{B}_{0}$ and we obtain

$$
-i \omega m_{e} \mathbf{B}_{0} \cdot \mathbf{v}=e\left[\mathbf{B}_{0} \cdot \mathbf{E}+\mathbf{B}_{0} \cdot\left(\mathbf{v} \times \mathbf{B}_{0}\right)\right]
$$

Since,

$$
\mathbf{B}_{0} \cdot\left(\mathbf{v} \times \mathbf{B}_{0}\right)=\mathbf{v} \cdot\left(\mathbf{B}_{0} \times \mathbf{B}_{0}\right)=\mathbf{0}
$$

Hence, substituting (5) into (6) we obtain

$$
-i \omega m_{e} \mathbf{B}_{0} \cdot \mathbf{v}=e \mathbf{B}_{0} \cdot \mathbf{E}
$$

which leads to

$$
\mathbf{B}_{0} \cdot \mathbf{v}=\frac{e}{-i \omega m_{e}} \mathbf{B}_{0} \cdot \mathbf{E}=\frac{i e}{\omega m_{e}} \mathbf{B}_{0} \cdot \mathbf{E}
$$

Substituting (8) into (4) we obtain

$$
-i \omega m_{e}\left(\mathbf{B}_{0} \times \mathbf{v}\right)=e\left[\mathbf{B}_{0} \times \mathbf{E}+\left|\mathbf{B}_{0}\right|^{2} \mathbf{v}-\frac{i e\left(\mathbf{B}_{0} \cdot \mathbf{E}\right) \mathbf{B}_{0}}{\omega m_{e}}\right]
$$

Next, we evaluate $\mathbf{B}_{0} \times \mathbf{v}$ as follows from (1)

$$
\mathbf{B}_{0} \times \mathbf{v}=-\mathbf{v} \times \mathbf{B}_{0}=\mathbf{E}+\frac{i \omega m_{e}}{e} \mathbf{v}
$$

Substituting (10) into (9) we obtain 
$-i \omega m_{e}\left(\mathbf{E}+\frac{i \omega m_{e}}{e} \mathbf{v}\right)=e\left[\mathbf{B}_{0} \times \mathbf{E}+\left|\mathbf{B}_{0}\right|^{2} \mathbf{v}-\frac{i e\left(\mathbf{B}_{0} \cdot \mathbf{E}\right) \mathbf{B}_{0}}{\omega m_{e}}\right]$

which is equivalent with

$\frac{\omega^{2} m_{e}{ }^{2}}{e} \mathbf{v}=i \omega m_{e} \mathbf{E}+e \mathbf{B}_{0} \times \mathbf{E}+e\left|\mathbf{B}_{0}\right|^{2} \mathbf{v}-\frac{i e^{2}\left(\mathbf{B}_{0} \cdot \mathbf{E}\right)}{\omega m_{e}} \mathbf{B}_{0}$

From ((6) of endnote II) we obtain the following vector form:

$$
\boldsymbol{\omega}_{B}=\frac{e \mathbf{B}_{0}}{m_{e}} \Rightarrow \mathbf{B}_{0}=\frac{m_{e}}{e} \boldsymbol{\omega}_{B}
$$

Substituting (13) into (11) yields

$$
\frac{\omega^{2} m_{e}{ }^{2} \mathbf{v}}{\mathrm{e}}-\frac{m_{e}{ }^{2}\left|\boldsymbol{\omega}_{B}\right|^{2} \mathbf{v}}{e}=m_{e}\left[i \omega \mathbf{E}+\boldsymbol{\omega}_{B} \times \mathbf{E}-\frac{i\left(\boldsymbol{\omega}_{B} \cdot \mathbf{E}\right) \boldsymbol{\omega}_{\mathrm{B}}}{\omega}\right]
$$

Which can be further written as

$$
\frac{\omega^{2} m_{e}}{e} \mathbf{v}-\frac{m_{e}}{e} \underbrace{\left|\boldsymbol{\omega}_{B}\right|^{2}}_{\omega_{B}{ }^{2}} \mathbf{v}=i \omega \mathbf{E}+\boldsymbol{\omega}_{B} \times \mathbf{E}-\frac{i\left(\boldsymbol{\omega}_{B} \cdot \mathbf{E}\right)}{\omega} \boldsymbol{\omega}_{B}
$$

Which produces the final result as

$$
\frac{m_{e}\left(\omega^{2}-\omega_{B}^{2}\right)}{e} \mathbf{v}=i \omega \mathbf{E}+\boldsymbol{\omega}_{\mathrm{B}} \times \mathbf{E}-\frac{i\left(\boldsymbol{\omega}_{\mathrm{B}} \cdot \mathbf{E}\right)}{\omega} \boldsymbol{\omega}_{\mathrm{B}}
$$

From ((3) of endnote II) we have

$$
\omega_{p}^{2}=\frac{e^{2} n_{e}}{\varepsilon_{0} m_{e}} \Rightarrow \frac{m_{e}}{e}=\frac{e n_{e}}{\varepsilon_{0} \omega_{p}^{2}}
$$

Substituting (17) into (16) we obtain:

$$
e n_{e} \frac{\left(\omega^{2}-\omega_{B}^{2}\right)}{\varepsilon_{0} \omega_{p}^{2}} \mathbf{v}=i \omega \mathbf{E}+\boldsymbol{\omega}_{B} \times \mathbf{E}-\frac{i\left(\boldsymbol{\omega}_{B} \cdot \mathbf{E}\right)}{\omega} \boldsymbol{\omega}_{B}
$$

Which is equivalent with

$$
e n_{e} \mathbf{v}=\frac{i \omega \varepsilon_{0} \omega_{p}^{2}}{\omega^{2}-\omega_{\mathrm{B}}^{2}} \mathbf{E}+\frac{\varepsilon_{0} \omega_{p}^{2} \boldsymbol{\omega}_{\mathrm{B}} \times \mathbf{E}}{\omega^{2}-\omega_{\mathrm{B}}^{2}}-\frac{i \varepsilon_{0} \omega_{p}^{2}\left(\boldsymbol{\omega}_{\mathrm{B}} \cdot \mathbf{E}\right) \boldsymbol{\omega}_{\mathrm{B}}}{\omega\left(\omega^{2}-\omega_{\mathrm{B}}^{2}\right)}
$$

Which can be further written as

$$
n_{e} e \mathbf{v}=-i \omega \varepsilon_{0}\left[\frac{-\omega_{p}^{2}}{\omega^{2}-\omega_{\mathrm{B}}{ }^{2}} \mathbf{E}+\frac{\omega_{p}^{2} \omega_{\mathrm{B}}\left(\boldsymbol{\omega}_{\mathrm{B}} \cdot \mathbf{E}\right)}{\omega^{2}\left(\omega^{2}-\omega_{\mathrm{B}}{ }^{2}\right)}+i \frac{\omega_{p}^{2} \boldsymbol{\omega}_{\mathrm{B}} \times \mathbf{E}}{\omega\left(\omega^{2}-\omega_{\mathrm{B}}{ }^{2}\right)}\right]
$$

Under the assumption that $\mathbf{B}_{0}$ is in the direction of the $Z$ axis let us find the permittivity ${ }^{\text {iv }}$ tensor $\boldsymbol{\varepsilon}$. From the second Maxwell equation we obtain

$$
\nabla \times \mathbf{H}=\underbrace{-i \omega \mathbf{D}_{\mathbf{0}}+\mathbf{J}_{\mathrm{p}}}_{-i \omega \mathbf{\varepsilon} \mathbf{E}}=-i \omega \underbrace{\mathbf{\varepsilon} \mathbf{E}}_{\mathbf{D}}
$$

Where

$$
\mathbf{D}_{\mathbf{0}}=\boldsymbol{\varepsilon}_{\mathbf{o}} \mathbf{E} \text { and } \mathbf{J}_{\mathrm{p}}=n_{e} e \mathbf{v}
$$

So, the permittivity tensor $\boldsymbol{\varepsilon}$ can be obtained from $-i \omega \mathbf{D}_{\mathbf{0}}+\mathbf{J}_{\mathrm{p}}=-i \omega \boldsymbol{\varepsilon} \mathbf{E} \Rightarrow-i \omega \boldsymbol{\varepsilon}_{\mathbf{o}} \mathbf{E}+n_{e} e \mathbf{v}=-i \omega \mathbf{\varepsilon} \mathbf{E}$

Which is equivalent with $\boldsymbol{\varepsilon}_{\mathbf{0}} \cdot \mathbf{E}+\frac{n_{e} e}{-i \omega} \mathbf{v}=\boldsymbol{\varepsilon} \cdot \mathbf{E} \Rightarrow \varepsilon_{\mathbf{o}} \mathbf{E}+\frac{i n_{e} e}{\omega} \mathbf{v}=\boldsymbol{\varepsilon} \mathbf{E}=\mathbf{D}$

Under the assumption that $\mathbf{B}_{0}$ is in the direction of the $z$ axis that means from (13) $\omega_{\mathrm{B}}$ is also in the direction of the $Z$ axis hence

$$
\boldsymbol{\omega}_{\mathrm{B}}\left(\boldsymbol{\omega}_{\mathrm{B}} \cdot \mathbf{E}\right)=E_{z} \omega_{\mathrm{B}}^{2} \mathbf{z} \& \boldsymbol{\omega}_{\mathrm{B}} \times \mathbf{E}=-\omega_{\mathrm{B}} E_{y} \mathbf{x}+\omega_{\mathrm{B}} E_{x} \mathbf{y}
$$

where $\mathbf{x}, \mathbf{y}$, and $\mathbf{z}$ is the unit vectors in the $x, y$, and $z$ direction respectively.

Substituting (25) into (20) we obtain

$$
\frac{n_{e} e}{-i \omega} \mathbf{v}=\frac{-\varepsilon_{0} \omega_{p}^{2}}{\omega^{2}-\omega_{\mathrm{B}}^{2}} \mathbf{E}+\frac{\varepsilon_{0} \omega_{p}^{2} E_{z} \omega_{\mathrm{B}}^{2} \mathbf{z}}{\omega^{2}\left(\omega^{2}-\omega_{\mathrm{B}}^{2}\right)}+i \frac{\varepsilon_{0} \omega_{p}^{2}\left(-\omega_{\mathrm{B}} E_{y} \mathbf{x}+\omega_{\mathrm{B}} E_{x} \mathbf{y}\right)}{\omega\left(\omega^{2}-\omega_{\mathrm{B}}{ }^{2}\right)}
$$

Which is equivalent with

$\frac{i n_{e} e}{\omega} \mathbf{v}=\frac{-\varepsilon_{0} \omega_{p}^{2}}{\omega^{2}-\omega_{B}^{2}} \mathbf{E}+\frac{\varepsilon_{0} \omega_{p}^{2} E_{z} \omega_{B}^{2} \mathbf{z}}{\omega^{2}\left(\omega^{2}-\omega_{\mathrm{B}}^{2}\right)}+i \frac{\varepsilon_{0} \omega_{p}^{2} \omega_{\mathrm{B}}\left(-E_{y} \mathbf{x}+E_{x} \mathbf{y}\right)}{\omega\left(\omega^{2}-\omega_{\mathrm{B}}^{2}\right)}$

or expanding (27) in components we obtain the matrix equation as follows

$\frac{i n_{e} e\left[\begin{array}{l}v_{x} \\ v_{y} \\ v_{z}\end{array}\right]}{\omega}=\frac{-\varepsilon_{0} \omega_{p}^{2}\left[\begin{array}{l}E_{x} \\ E_{y} \\ E_{Z}\end{array}\right]}{\omega^{2}-\omega_{\mathrm{B}}{ }^{2}}+\frac{\varepsilon_{0} \omega_{p}^{2} \omega_{\mathrm{B}}^{2}\left[\begin{array}{c}0 \\ 0 \\ E_{z}\end{array}\right]}{\omega^{2}\left(\omega^{2}-\omega_{\mathrm{B}}^{2}\right)}-\frac{i \varepsilon_{0} \omega_{p}^{2} \omega_{\mathrm{B}}\left[\begin{array}{ccc}0 & 1 & 0 \\ -1 & 0 & 0 \\ 0 & 0 & 0\end{array}\right]\left[\begin{array}{c}E_{x} \\ E_{y} \\ E_{\mathrm{Z}}\end{array}\right]}{\omega\left(\omega^{2}-\omega_{\mathrm{B}}{ }^{2}\right)}$

Substituting (28) into (24) we obtain the permittivity tensor $\boldsymbol{\varepsilon}$ as follows:

$$
\left[\begin{array}{ccc}
\varepsilon & -i \varepsilon_{g} & 0 \\
i \varepsilon_{g} & \varepsilon & 0 \\
0 & 0 & \varepsilon_{z}
\end{array}\right]=\varepsilon
$$

where

$$
\begin{aligned}
& \varepsilon=\varepsilon_{o}\left(1-\frac{\omega_{p}^{2}}{\omega^{2}-\omega_{\mathrm{B}}^{2}}\right) \\
& \varepsilon_{g}=\frac{\varepsilon_{0} \omega_{p}^{2} \omega_{\mathrm{B}}}{\omega\left(\omega^{2}-\omega_{\mathrm{B}}^{2}\right)}
\end{aligned}
$$

$$
\varepsilon_{z}=\varepsilon_{o}\left[1-\frac{\omega_{p}^{2}}{\omega^{2}-\omega_{\mathrm{B}}^{2}}+\frac{\omega_{p}^{2} \omega_{\mathrm{B}}^{2}}{\omega^{2}\left(\omega^{2}-\omega_{\mathrm{B}}^{2}\right)} \equiv 1-\frac{\omega_{p}^{2}\left(1-\frac{\omega_{\mathrm{B}}^{2}}{\omega^{2}}\right)}{\omega^{2}-\omega_{\mathrm{B}}^{2}}\right]
$$

Which is equivalent with

$$
\varepsilon_{z}=\varepsilon_{o}\left[1-\frac{\omega_{p}^{2}}{\omega^{2}-\omega_{\mathrm{B}}^{2}}+\frac{\omega_{p}^{2} \omega_{\mathrm{B}}^{2}}{\omega^{2}\left(\omega^{2}-\omega_{\mathrm{B}}^{2}\right)} \equiv 1-\frac{\omega_{p}^{2}}{\omega^{2}}\right]
$$

If we make the substitutions made by in Jandieri et al, 2007 [14] pg. 309

$$
v=\frac{\omega_{p}^{2}}{\omega^{2}} \text { and } u=\frac{\omega_{\mathrm{B}}^{2}}{\omega^{2}}
$$

then (29)-(33) becomes 


$$
\begin{aligned}
& \varepsilon=\varepsilon_{o}\left(1-\frac{v}{1-u}\right) \\
& \varepsilon_{g}=\varepsilon_{0} \frac{v \sqrt{u}}{1-u} \\
& \varepsilon_{z}=\varepsilon_{o}(1-v)
\end{aligned}
$$

Let us compare now (35)-(37) with the results of (1) in Jandieri et al, 2007 [14] pg. 309 in (cgs units).

$$
\begin{aligned}
& \varepsilon=1-\left.\frac{v(1-i s)}{(1-i s)^{2}-u} v\right|_{s=0}=1-\frac{v}{1-u} \\
& \varepsilon_{g}=\left.\frac{v \sqrt{u}}{(1-i s)^{2}-u}\right|_{s=0}=\frac{v \sqrt{u}}{1-u} \\
& \varepsilon_{z}=1-\left.\frac{v}{1-i s}\right|_{s=0}=1-v
\end{aligned}
$$

Since, (38)-(40) for $s=0$ in (cgs units) is the same as (35)(37) in (SI units) it is very likely that (38)-(40) might be OK for the general case of $s \neq 0$; however, there is no guaranty that (38)-(40) are OK. So, we must prove that (38)-(40) are accurate descriptions of the permittivity tensor $\boldsymbol{\varepsilon}$ (cgs units) for the general case of $s \neq 0$.

Next, we need to find the refractive index, $n$; however, before we can do that we need to find the impermittivity ${ }^{\mathrm{vi}}$ tensor, $\boldsymbol{\kappa}[9]$ :

$\mathbf{E}=\boldsymbol{\kappa} \cdot \mathbf{D}$

Substituting (24) into (41) we obtain the impermittivity tensor, к [9]:

$\mathbf{E}=\boldsymbol{\kappa} \cdot \underbrace{\boldsymbol{\varepsilon} \cdot \mathbf{E}}_{\mathbf{D}} \Rightarrow \boldsymbol{\kappa} \cdot \boldsymbol{\varepsilon}=\mathbf{I} \Rightarrow \boldsymbol{\kappa}=\boldsymbol{\varepsilon}^{-1}$

$\boldsymbol{\kappa}=\boldsymbol{\varepsilon}^{-1}=\left[\begin{array}{lll}\kappa_{11} & \kappa_{12} & \kappa_{13} \\ \kappa_{21} & \kappa_{22} & \kappa_{23} \\ \kappa_{31} & \kappa_{32} & \kappa_{33}\end{array}\right]=\frac{\operatorname{adj}(\boldsymbol{\varepsilon})}{|\boldsymbol{\varepsilon}| \equiv \operatorname{det}(\boldsymbol{\varepsilon})}$

$\operatorname{adj}(\boldsymbol{\varepsilon})=\left[\begin{array}{ccc}\varepsilon \varepsilon_{z} & i \varepsilon_{g} \varepsilon_{z} & 0 \\ -i \varepsilon_{g} \varepsilon_{z} & \varepsilon \varepsilon_{z} & 0 \\ 0 & 0 & \varepsilon^{2}-\varepsilon_{g}^{2}\end{array}\right]$

$|\varepsilon|=\varepsilon_{z}\left(\varepsilon^{2}-\varepsilon_{g}{ }^{2}\right)$

Substituting (44) and (45) into (43) yields

$\boldsymbol{\kappa}=\boldsymbol{\varepsilon}^{-1}=\left[\begin{array}{ccc}\kappa & i \kappa_{g} & 0 \\ -i \kappa_{g} & \kappa & 0 \\ 0 & 0 & \kappa_{z}\end{array}\right]$ vii
Where

$$
\begin{aligned}
& \kappa=\frac{\varepsilon \varepsilon_{z}}{\varepsilon_{z}\left(\varepsilon^{2}-\varepsilon_{g}^{2}\right)}=\frac{\varepsilon}{\varepsilon^{2}-\varepsilon_{g}^{2}} \\
& \kappa_{g}=\frac{\varepsilon_{g} \varepsilon_{z}}{\varepsilon_{z}\left(\varepsilon^{2}-\varepsilon_{g}^{2}\right)}=\frac{\varepsilon_{g}}{\varepsilon^{2}-\varepsilon_{g}^{2}} \\
& \kappa_{g}=\frac{\varepsilon^{2}-\varepsilon_{g}^{2}}{\varepsilon_{z}\left(\varepsilon^{2}-\varepsilon_{g}^{2}\right)}=\frac{1}{\varepsilon_{z}}
\end{aligned}
$$

Substituting (47)-(49) into (46) yields

$$
\boldsymbol{\kappa}=\boldsymbol{\varepsilon}^{-1}=\left[\begin{array}{ccc}
\kappa & i \kappa_{g} & 0 \\
-i \kappa_{g} & \kappa & 0 \\
0 & 0 & \kappa_{z}
\end{array}\right]=\left[\begin{array}{ccc}
\frac{\varepsilon}{\varepsilon^{2}-\varepsilon_{g}^{2}} & \frac{i \varepsilon_{g}}{\varepsilon^{2}-\varepsilon_{g}{ }^{2}} & 0 \\
\frac{-i \varepsilon_{g}}{\varepsilon^{2}-\varepsilon_{g}^{2}} & \frac{\varepsilon}{\varepsilon^{2}-\varepsilon_{g}^{2}} & 0 \\
0 & 0 & \frac{1}{\varepsilon_{z}}
\end{array}\right]
$$

where the impermittivity coefficients $\kappa, \kappa_{g}$, and $\kappa_{z}$ are obtained from

$$
\kappa=\frac{\varepsilon}{\varepsilon^{2}-\varepsilon g^{2}}=\frac{1}{\varepsilon_{0}} \frac{1-\frac{v}{1-u}}{\left(1-\frac{v}{1-u}\right)^{2}-\left(\frac{v \sqrt{u}}{1-u}\right)^{2}}
$$

$$
\begin{aligned}
& =\frac{1}{\varepsilon_{0}} \frac{(1-u)(1-u-v)}{\left[(1-u-v)^{2}-v^{2} u\right]}=\frac{1}{\varepsilon_{0}} \frac{(1-u)(1-u-v)}{\left[(1-u)^{2}-2(1-u) v+v^{2}-v^{2} u\right]} \\
& =\frac{1}{\varepsilon_{0}}\left[\frac{(1-u)(1-u-v)}{\left[(1-u)^{2}-2(1-u) v+v^{2}(1-u)\right]} \equiv \frac{1-v-u}{1-2 v+v^{2}-u}\right]
\end{aligned}
$$

Which can be further written as

$\kappa=\frac{1}{\varepsilon_{0}} \frac{1-v-u}{(1-v)^{2}-u}$

Next, we compute $\kappa_{g}$ as follows

$$
\kappa_{g}=\frac{\varepsilon_{g}}{\varepsilon^{2}-\varepsilon^{2}}=\frac{1}{\varepsilon_{0}} \frac{\frac{v \sqrt{u}}{1-u}}{\left(1-\frac{v}{1-u}\right)^{2}-\left(\frac{v \sqrt{u}}{1-u}\right)^{2}}
$$

$$
\begin{aligned}
& =\frac{1}{\varepsilon_{0}} \frac{(1-u)(v \sqrt{u})}{\left[(1-u-v)^{2}-v^{2} u\right]}=\frac{1}{\varepsilon_{0}} \frac{(1-u)(v \sqrt{u})}{\left[(1-u)^{2}-2(1-u) v+v^{2}-v^{2} u\right]} \\
& =\frac{1}{\varepsilon_{0}}\left[\frac{(1-u)(v \sqrt{u})}{\left[(1-u)^{2}-2(1-u) v+v^{2}(1-u)\right]} \equiv \frac{v \sqrt{u}}{1-2 v+v^{2}-u}\right]
\end{aligned}
$$

Which can be further written as

$\kappa_{g}=\frac{1}{\varepsilon_{0}} \frac{v \sqrt{u}}{(1-v)^{2}-u}$

Next, we compute $\kappa_{z}$ as follows

$\kappa_{z}=\frac{1}{\varepsilon_{z}}=\frac{1}{\varepsilon_{0}} \frac{1}{1-v}$ 
The dispersion relation in the $k D B$ system Kong, 1990 [9] pg. $76(5)$ is given by

$\mathbf{K} \cdot\left[\begin{array}{l}D_{1} \\ D_{2}\end{array}\right]=\mathbf{W} \cdot\left[\begin{array}{l}B_{1} \\ B_{2}\end{array}\right]$

And

$v\left[\begin{array}{l}B_{1} \\ B_{2}\end{array}\right]=\left[\begin{array}{cc}0 & w \\ -w & 0\end{array}\right] \cdot\left[\begin{array}{l}D_{1} \\ D_{2}\end{array}\right]$

Where

$\mathbf{K}=\left[\begin{array}{cc}\kappa & i \kappa_{g} \cos \theta \\ -i \kappa_{g} \cos \theta & \kappa \cos ^{2} \theta+\kappa_{z} \sin ^{2} \theta\end{array}\right]$

And

$\mathbf{W}=\left[\begin{array}{cc}0 & w \\ -w & 0\end{array}\right]$

Eliminating $B_{k}$ yields

$\left[\begin{array}{cc}w^{2}-v \kappa & -i v \kappa_{g} \cos \theta \\ i v \kappa_{g} \cos \theta & w^{2}-v\left(\kappa \cos ^{2} \theta+\kappa_{z} \sin ^{2} \theta\right)\end{array}\right] \cdot\left[\begin{array}{l}D_{1} \\ D_{2}\end{array}\right]=0$

For non-trivial solutions for $D_{k}$ we set determinant of the $2 \times 2$ equal to zero and obtain

$w^{2}=\frac{v\left[\kappa\left(1+\cos ^{2} \theta\right)+\kappa_{z} \sin ^{2} \theta \pm \sqrt{\left(\kappa-\kappa_{z}\right)^{2} \sin ^{4} \theta+4 \kappa_{g}^{2} \cos ^{2} \theta}\right]}{2}$

The refractive index is defined as the ratio Kong, 1990 [9] top of pg. 78

$n^{2}=\frac{D_{1}}{\varepsilon_{0} E_{1}}=\frac{D_{2}}{\varepsilon_{0} E_{2}}$

where

$\left[\begin{array}{cc}\kappa & i \kappa_{g} \cos \theta \\ -i \kappa_{g} \cos \theta & \kappa \cos ^{2} \theta+\kappa_{z} \sin ^{2} \theta\end{array}\right] \cdot\left[\begin{array}{c}D_{1} \\ D_{2}\end{array}\right]=\left[\begin{array}{c}E_{1} \\ E_{2}\end{array}\right]$

From (61) we find that

$w^{2} D_{1}=v\left(\kappa \mathrm{D}_{1}+\mathrm{i} \kappa_{g} \cos \theta D_{2}\right)=v E_{1}$

Hence, substituting (64) and (61) into (62) we obtain:

$$
n^{2}=\frac{v E_{1}}{\varepsilon_{0} w^{2} E_{1}}=\frac{v}{\varepsilon_{0} w^{2}}
$$

Which is equivalent with

$$
n^{2}=\frac{w^{2}}{\varepsilon_{0} w^{2}} \frac{2}{\kappa\left(1+\cos ^{2} \theta\right)+\kappa_{Z} \sin ^{2} \theta \pm \sqrt{\left(\kappa-\kappa_{Z}\right)^{2} \sin ^{4} \theta+4 \kappa_{g}^{2} \cos ^{2} \theta}}
$$

Which is equivalent with

$n^{2}=\frac{v E_{2}}{\varepsilon_{0} w^{2} E_{2}}=\frac{v}{\varepsilon_{0} w^{2}}$ viii

Hence, the refractive index squared, $n^{2}$, can be obtained from

$n^{2}=\frac{1}{\varepsilon_{0}} \frac{2}{\kappa\left(1+\cos ^{2} \theta\right)+\kappa_{z} \sin ^{2} \theta \pm \sqrt{\left(\kappa-\kappa_{z}\right)^{2} \sin ^{4} \theta+4 \kappa_{g}^{2} \cos ^{2} \theta}}$ ix

Which can be written in compact form as

$n^{2}=\frac{1}{\varepsilon_{0}} \frac{2}{x \pm \sqrt{y}}$

Where

$x=\kappa\left(1+\cos ^{2} \theta\right)+\kappa_{z} \sin ^{2} \theta$

And

$y=\left(\kappa-\kappa_{z}\right)^{2} \sin ^{4} \theta+4 \kappa_{g}{ }^{2} \cos ^{2} \theta$

Next, substituting the values of $\kappa(52), \kappa_{g},(54)$ and $\kappa_{z}(55)$ into (70) and (71) the following expression of the refractive index squared is obtained.

$x=\frac{1}{\varepsilon_{0}} \frac{\vartheta-u}{\vartheta^{2}-u}\left(1+\cos ^{2} \theta\right)+\frac{1}{\varepsilon_{0}} \frac{1}{\vartheta} \sin ^{2} \theta$

And

$y=\left(\frac{1}{\varepsilon_{0}} \frac{\vartheta-u}{\vartheta^{2}-u}-\frac{1}{\varepsilon_{0}} \frac{1}{\vartheta}\right)^{2} \sin ^{4} \theta+4\left(\frac{1}{\varepsilon_{0}} \frac{v \sqrt{u}}{\vartheta^{2}-u}\right)^{2} \cos ^{2} \theta$

Where

$\vartheta \equiv 1-v$

which is equivalent with (see Appendix A)

$n^{2}=\frac{2\left(\vartheta^{2}-u\right) \vartheta}{2\left(\vartheta^{2}-u\right)+u v\left(1+\cos ^{2} \theta\right) \pm v \sqrt{u^{2} \sin ^{4} \theta+4 \vartheta^{2} u \cos ^{2} \theta}} \times$

Next, let us derive the simplified formula

$$
n^{2}=1-\left[1-\frac{2\left(\vartheta^{2}-u\right) \vartheta}{2\left(\vartheta^{2}-u\right)+u v\left(1+\cos ^{2} \theta\right) \pm v \sqrt{u^{2} \sin ^{4} \theta+4 \vartheta^{2} u \cos ^{2} \theta}}\right]
$$

$$
=1-v \frac{2 \vartheta^{2}-u \sin ^{2} \theta \pm \sqrt{u^{2} \sin ^{4} \theta+4 \vartheta^{2} u \cos ^{2} \theta}}{2\left(\vartheta^{2}-u\right)+u v\left(1+\cos ^{2} \theta\right) \pm v \sqrt{u^{2} \sin ^{4} \theta+4 \vartheta^{2} u \cos ^{2} \theta}}
$$

Or, by taking the square root, the following closed form expression of the refractive index is obtained

$n=\sqrt{1-\frac{v\left[2 \vartheta^{2}-u \sin ^{2} \theta \pm \sqrt{u^{2} \sin ^{4} \theta+4 \vartheta^{2} u \cos ^{2} \theta}\right]}{2\left(\vartheta^{2}-u\right)+u v\left(1+\cos ^{2} \theta\right) \pm v \sqrt{u^{2} \sin ^{4} \theta+4 \vartheta^{2} u \cos ^{2} \theta}}}$

A formula (see Appendix B) is used which is known as the Appleton-Hartree magneto-ionic formula [2] derived from polarization-current arguments Kong, 1990 pg. 78 (14) [9] as follows 
$n^{2}=1-v \frac{2 \vartheta}{2 \vartheta-u \sin ^{2} \theta \mp \sqrt{u^{2} \sin ^{4} \theta+4 \vartheta^{2} u \cos ^{2} \theta}}$

Which is identical to

$n=\sqrt{1-v \frac{2 \vartheta}{2 \vartheta-u \sin ^{2} \theta \mp \sqrt{u^{2} \sin ^{4} \theta+4 \vartheta^{2} u \cos ^{2} \theta}}}$ xi

This concludes the derivations of the refractive index in anisotropic absorptive particle collision-less magnetized plasma. Next, we discuss the refractive index in anisotropic absorptive turbulent magnetized plasma which includes the effect of collisions.

\section{Refractive Index in Anisotropic Absorptive Turbulent Magnetized Plasma}

Perhaps a more realistic stationary example is that of the refractive index in anisotropic absorptive turbulent magnetized plasma whose simplest model of collision is as follows.

What is Ionosphere absorption? How do we model it?

Ionosphere absorption is of critical importance when designing a GNSS or an RF signal, particularly when trying to determine propagation conditions to study the refractive index [23]. When an RF signal coming from an RF emitter (typically a GPS, or GNSS satellite) propagates through the ionosphere, the receiver down on the Earth receives the refracted portion of the signal. As a result of this refraction, which is enables the long-distance propagation of radio waves; some of the shortwave signal strength is decreased. In this regard, ISAB is the primary limiting factor in radio propagation [23].

What is the mechanism of the ISAB? ISAB is only a factor in the period of the day where radio signals travel through the portion of the ionosphere facing the sun. The solar wind and radiation cause the ionosphere to become charged with electrons in the first place. At night, the atmosphere becomes drained of its charge, and radio signals can go much farther with less loss of signal. In particular, low frequency signals that would be attenuated to nothing during the day will be received much farther away at night [23].

Consider an electron plasma with collisions and introduce a collision frequency xii $\quad \omega_{\text {eff }}=2 \pi v_{\text {eff }} \approx 2 \pi n_{e} T^{-1.5} \quad$ (see Appendix C) such that a damping factor is introduced and the force on the electron in the $x, y$, and $z$-direction becomes

$\mathbf{F}=\frac{d\left(m_{e} \mathbf{v}\right)}{d t}+\omega_{\mathrm{eff}} m_{e} \mathbf{v}$

So, now we repeat (1) through (40). Hence, substituting (80) into (1), (1) becomes $\left(\omega_{\text {eff }}-i \omega \equiv \varpi\right) m_{e} \mathbf{v}=e\left[\mathbf{E}+\mathbf{v} \times \mathbf{B}_{0}\right]$

Let us apply the cross product into (81) so we obtain:

$\varpi m_{e}\left(\mathbf{B}_{0} \times \mathbf{v}\right)=e\left[\mathbf{B}_{0} \times \mathbf{E}+\underline{\mathbf{B}_{0} \times\left(\mathbf{v} \times \mathbf{B}_{0}\right)}\right]$

Substituting (3) and (8) into (82) produces

$\varpi m_{e}\left(\mathbf{B}_{0} \times \mathbf{v}\right)=e\left[\mathbf{B}_{0} \times \mathbf{E}+\left|\mathbf{B}_{0}\right|^{2} \mathbf{v}-\frac{i e\left(\mathbf{B}_{0} \cdot \mathbf{E}\right) \mathbf{B}_{0}}{\omega m_{e}}\right]$

Rewriting (83) we obtain

$\mathbf{B}_{0} \times \mathbf{v}=-\mathbf{v} \times \mathbf{B}_{0}=\mathbf{E}-\varpi m_{e} e^{-1} \mathbf{v}$

Substituting (84) into (83) we obtain

$m_{e} \varpi\left(\mathbf{E}-\frac{\varpi m_{e}}{e} \mathbf{v}\right)=e\left[\mathbf{B}_{0} \times \mathbf{E}+\left|\mathbf{B}_{0}\right|^{2} \mathbf{v}-\frac{i e\left(\mathbf{B}_{0} \cdot \mathbf{E}\right)}{\omega m_{e}} \mathbf{B}_{0}\right]$

$\frac{\varpi^{2} m_{e}^{2}}{e} \mathbf{v}=\varpi m_{e} \mathbf{E}-e \mathbf{B}_{0} \times \mathbf{E}-e\left|\mathbf{B}_{0}\right|^{2} \mathbf{v}+\frac{i e^{2}\left(\mathbf{B}_{0} \cdot \mathbf{E}\right)}{\omega m_{e}} \mathbf{B}_{0}$

Substituting (13) into (86) we obtain

$\frac{\varpi^{2} m_{e}}{e} \mathbf{v}+\frac{m_{e}}{e} \underbrace{\left|\boldsymbol{\omega}_{\mathrm{B}}\right|^{2}}_{\omega_{\mathrm{B}}{ }^{2}} \mathbf{v}=\varpi \mathbf{E}-\boldsymbol{\omega}_{\mathrm{B}} \times \mathbf{E}+\frac{i\left(\boldsymbol{\omega}_{\mathrm{B}} \cdot \mathbf{E}\right)}{\omega} \boldsymbol{\omega}_{\mathrm{B}}$

Which is identical to

$\frac{m_{e}\left(\varpi^{2}+\omega_{B}^{2}\right)}{e} \mathbf{v}=\varpi \mathbf{E}-\boldsymbol{\omega}_{\mathrm{B}} \times \mathbf{E}+\frac{i\left(\boldsymbol{\omega}_{\mathrm{B}} \cdot \mathbf{E}\right)}{\omega} \boldsymbol{\omega}_{\mathrm{B}}$

Substituting (17) into (87) we obtain:

$e n_{e} \frac{\left[\varpi^{2}+\omega_{B}^{2}\right]}{\varepsilon_{0} \omega_{p}^{2}} \mathbf{v}=\varpi \mathbf{E}-\boldsymbol{\omega}_{\mathrm{B}} \times \mathbf{E}+\frac{i\left(\boldsymbol{\omega}_{\mathrm{B}} \cdot \mathbf{E}\right)}{\omega} \boldsymbol{\omega}_{\mathrm{B}}$

Which is equivalent with

$e n_{e} \mathbf{v}=\frac{\varpi \varepsilon_{0} \omega_{p}^{2}}{\varpi^{2}+\omega_{\mathrm{B}}^{2}} \mathbf{E}-\frac{\varepsilon_{0} \omega_{p}^{2} \boldsymbol{\omega}_{\mathrm{B}} \times \mathbf{E}}{\varpi^{2}+\omega_{\mathrm{B}}{ }^{2}}+\frac{i \varepsilon_{0} \omega_{p}^{2}\left(\boldsymbol{\omega}_{\mathrm{B}} \cdot \mathbf{E}\right)}{\omega\left(\varpi^{2}+\omega_{B}{ }^{2}\right)} \boldsymbol{\omega}_{\mathrm{B}}$

Substituting (25) into (90) we obtain

$n_{e} e \mathbf{v}=\frac{\varpi \varepsilon_{0} \omega_{p}^{2}}{\varpi^{2}+\omega_{\mathrm{B}}^{2}} \mathbf{E}+\frac{i \varepsilon_{0} \omega_{p}^{2} E_{z} \omega_{B}^{2} \mathbf{z}}{\omega\left(\varpi^{2}+\omega_{\mathrm{B}}^{2}\right)}-\frac{i \varepsilon_{0} \omega_{p}^{2} \omega_{\mathrm{B}}\left(-E_{y} \mathbf{x}+E_{x} \mathbf{y}\right)}{\varpi^{2}+\omega_{\mathrm{B}}^{2}}$

Expanding (91) in components we obtain the following matrix equation

$n_{e} e\left[\begin{array}{l}v_{x} \\ v_{y} \\ v_{z}\end{array}\right]=\frac{\varpi \varepsilon_{0} \omega_{p}^{2}\left[\begin{array}{l}E_{x} \\ E_{y} \\ E_{z}\end{array}\right]+\frac{i \varepsilon_{0} \omega_{p}^{2} \omega_{B}^{2}}{\omega}\left[\begin{array}{c}0 \\ 0 \\ E_{z}\end{array}\right]-i \varepsilon_{0} \omega_{p}^{2} \omega_{B}\left[\begin{array}{ccc}0 & 1 & 0 \\ -1 & 0 & 0 \\ 0 & 0 & 0\end{array}\right]\left[\begin{array}{l}E_{x} \\ E_{y} \\ E_{z}\end{array}\right]}{\varpi^{2}+\omega_{B}{ }^{2}}(92)$

Substituting (91) into (24) we obtain the permittivity tensor $\boldsymbol{\varepsilon}$ as follows:

$$
\boldsymbol{\varepsilon}=\left[\begin{array}{ccc}
\varepsilon & -i \varepsilon_{g} & 0 \\
i \varepsilon_{g} & \varepsilon & 0 \\
0 & 0 & \varepsilon_{z}
\end{array}\right]
$$

where 


$$
\begin{aligned}
& \varepsilon=\varepsilon_{o}\left\{1+\left[\frac{i \varpi \omega_{p}^{2}}{\omega\left(\varpi^{2}+\omega_{B}^{2}\right)} \equiv \frac{-\varpi \omega_{p}^{2}}{\omega\left(\varpi^{2}-\omega_{B}^{2}\right)}\right]\right\} \\
& \varepsilon_{g}=\varepsilon_{o}\left[\frac{-\omega_{p}^{2} \omega_{B}}{\omega\left(\varpi^{2}+\omega_{B}^{2}\right)} \equiv \frac{\omega_{p}^{2} \omega_{B}}{\omega\left(\varpi^{2}-\omega_{B}^{2}\right)}\right] \\
& \varepsilon_{Z}=\varepsilon_{o}\left[1+\frac{i \varpi \omega_{p}^{2}}{\omega\left(\varpi^{2}+\omega_{B}^{2}\right)}-\frac{\omega_{p}^{2} \omega_{B}^{2}}{\omega^{2}\left(\varpi^{2}+\omega_{B}^{2}\right)}\right]
\end{aligned}
$$

It is straightforward to show that (94) through (96) are identical to

$$
\begin{aligned}
& \varepsilon=\varepsilon_{o}\left[1-\frac{\left(1+i \frac{\omega_{\mathrm{eff}}}{\omega}\right) \frac{\omega_{p}^{2}}{\omega^{2}}}{\left(1+i \frac{\omega_{\mathrm{eff}}}{\omega}\right)^{2}-\frac{\omega_{B}{ }^{2}}{\omega^{2}}}\right] \\
& \varepsilon_{g}=\varepsilon_{o}\left[\frac{\frac{\omega_{p}^{2} \omega_{B}}{\omega^{2} \omega}}{\left(1+i \frac{\omega_{\mathrm{eff}}}{\omega}\right)^{2}-\frac{\omega_{B}{ }^{2}}{\omega^{2}}}\right] \\
& \varepsilon_{z}=\varepsilon_{o}\left[1-\frac{\frac{\omega_{p}^{2}}{\omega^{2}}\left(1+i \frac{\omega_{\mathrm{eff}}}{\omega}-\frac{\omega_{B}{ }^{2}}{\omega^{2}}\right)}{\left(1+i \frac{\omega_{\mathrm{eff}}}{\omega}\right)^{2}-\frac{\omega_{B}{ }^{2}}{\omega^{2}}}\right]
\end{aligned}
$$

Next, if we make the substitutions (31) made by in Jandieri et al, 2007 [14] pg. 309

$$
\begin{aligned}
& \varepsilon=\varepsilon_{o}\left[1-\frac{v(1+i s)}{(1+i s)^{2}-u}\right] \\
& \varepsilon_{g}=\varepsilon_{o}\left[\frac{v \sqrt{u}}{(1+i s)^{2}-u}\right] \\
& \varepsilon_{z}=\varepsilon_{o}\left[1-\frac{v(1+i s-u)}{(1+i s)^{2}-u}\right]
\end{aligned}
$$

Where

$$
s=\frac{\omega_{\mathrm{eff}}}{\omega}
$$

We obtain the permittivity tensor $\boldsymbol{\varepsilon}$ in compact form (see (29)).

Let us compare now (100)-(102) with the results of (1) in Jandieri et al, 2007 [14] pg. 309 in (cgs units).

$$
\begin{aligned}
& \varepsilon=1-\frac{v(1-i s)}{(1-i s)^{2}-u} \text { xiii } \\
& \varepsilon_{g}=\frac{v \sqrt{u}}{(1-i s)^{2}-u} \\
& \varepsilon_{z}=1-\frac{v}{1-i s}
\end{aligned}
$$

Clearly, it shows that Jandieri et al, 2007 [14] has made a few unknown assumptions which we need to explain them further in Appendix D.

Similarly, from (50) let us find the impermittivity tensor $\boldsymbol{\kappa}$ components as follows

$$
\boldsymbol{\kappa}=\boldsymbol{\varepsilon}^{-1}=\left[\begin{array}{ccc}
\kappa \equiv \frac{\varepsilon}{\varepsilon^{2}-\varepsilon_{g}^{2}} & i\left(\kappa_{g} \equiv \frac{\varepsilon_{g}}{\varepsilon^{2}-\varepsilon_{g}^{2}}\right) & 0 \\
-i \kappa_{g} & \kappa & 0 \\
0 & 0 & \kappa_{z} \equiv \frac{1}{\varepsilon_{z}}
\end{array}\right]
$$

where

$$
\begin{aligned}
& \kappa=\frac{\varepsilon}{\varepsilon^{2}-\varepsilon_{g}^{2}}=\frac{1}{\varepsilon_{0}} \frac{1-\frac{v(1+i s)}{(1+i s)^{2}-u}}{\left[1-\frac{v(1+i s)}{(1+i s)^{2}-u}\right]^{2}-\left[\frac{v \sqrt{u}}{(1+i s)^{2}-u}\right]^{2}} \\
& \kappa_{g}=\frac{\varepsilon_{g}}{\varepsilon^{2}-\varepsilon_{g}^{2}}=\frac{1}{\varepsilon_{0}} \frac{\frac{v \sqrt{u}}{(1+i s)^{2}-u}}{\left[1-\frac{v(1-i s)}{(1+i s)^{2}-u}\right]^{2}-\left[\frac{v \sqrt{u}}{(1+i s)^{2}-u}\right]^{2}} \\
& \kappa_{z}=\frac{1}{\varepsilon_{z}}=\frac{1}{\varepsilon_{0}} \frac{1}{1-\frac{v(1+i s-u)}{\left[(1+i s)^{2}-u\right]}}
\end{aligned}
$$

Performing simplification calculations with complex numbers (see Appendix E) (108) through (110) are identical to,

$$
\begin{gathered}
\kappa=\frac{1}{\varepsilon_{0}} \frac{(1+i s)^{2}-v(1+i s)-u \equiv a}{[(1+i s)-v]^{2}-u \equiv d} \\
\kappa_{g}=\frac{1}{\varepsilon_{0}} \frac{v \sqrt{u}}{[(1+i s)-v]^{2}-u} \\
\kappa_{z}=\frac{1}{\varepsilon_{0}} \frac{(1+i s)^{2}-u}{(1+i s)^{2}-v(1+i s)-u+v u \equiv b}
\end{gathered}
$$

Let us find now the refractive index from (68) as follows:

$$
n^{2}=\frac{1}{\varepsilon_{0}} \frac{2}{\kappa\left(1+\cos ^{2} \theta\right)+\kappa_{Z} \sin ^{2} \theta \pm \sqrt{\left(\kappa-\kappa_{Z}\right)^{2} \sin ^{4} \theta+4 \kappa_{g}^{2} \cos ^{2} \theta}}
$$

In Appendix $\mathrm{F}$ we perform the substitution of $\kappa, \kappa_{g}$, and $\kappa_{z}$ given by (111) through (113) in (114) and after some algebra we obtain the following

$$
n^{2}=1-\frac{2(a-d) b-v u c \sin ^{2} \theta \pm v \sqrt{u^{2} c^{2} \sin ^{4} \theta+4 u b^{2} \cos ^{2} \theta}}{2 a b-v u c \sin ^{2} \theta \pm v \sqrt{u^{2} c^{2} \sin ^{4} \theta+4 u b^{2} \cos ^{2} \theta}}
$$

Which is identical to

$$
n^{2}=1-v \frac{2 q b-u c \sin ^{2} \theta \pm \sqrt{u^{2} c^{2} \sin ^{4} \theta+4 u b^{2} \cos ^{2} \theta}}{2 a b-v u c \sin ^{2} \theta \pm v \sqrt{u^{2} c^{2} \sin ^{4} \theta+4 u b^{2} \cos ^{2} \theta}}
$$

A formula (see Appendix G) is used which is known as the Appleton-Hartree magneto-ionic formula derived from polarization-current arguments as follows

$$
1-n^{2}=v \frac{2\left(b-i s u \sin ^{2} \theta\right)}{2 f+u g \sin ^{2} \theta \mp \sqrt{u^{2} c^{2} \sin ^{4} \theta+4 u b^{2} \cos ^{2} \theta}} \text { xiv }
$$




$$
n^{2}=\sqrt{1-v \frac{2\left(b-i s u \sin ^{2} \theta\right)}{2 f+u g \sin ^{2} \theta \mp \sqrt{u^{2} c^{2} \sin ^{4} \theta+4 u b^{2} \cos ^{2} \theta}}} \mathrm{xv}
$$

where $f$ and $g$ are defined in Appendix G. Finally, in Appendix H, we discuss special cases.

This concludes the derivations of the refractive index in anisotropic absorptive particle magnetized plasma.

\section{Numerical, Theoretical Results}

In this section we provide numerical theoretical results on the refractive index in anisotropic absorptive particle collision-less magnetized plasma and refractive index in anisotropic absorptive turbulent magnetized plasma.

\subsection{Refractive Index in Anisotropic Absorptive Particle Collision-less Magnetized Plasma}

In this subsection we present, numerical results of refractive index and of the extinction coefficient using the (68)-(79) (i.e., the collision frequency is assumed to be zero).

Typically, at night the ionosphere is not disturbed by the Sun. Furthermore, assuming that there are not space vehicles traveling in the vicinity of the path of the signal of propagation.
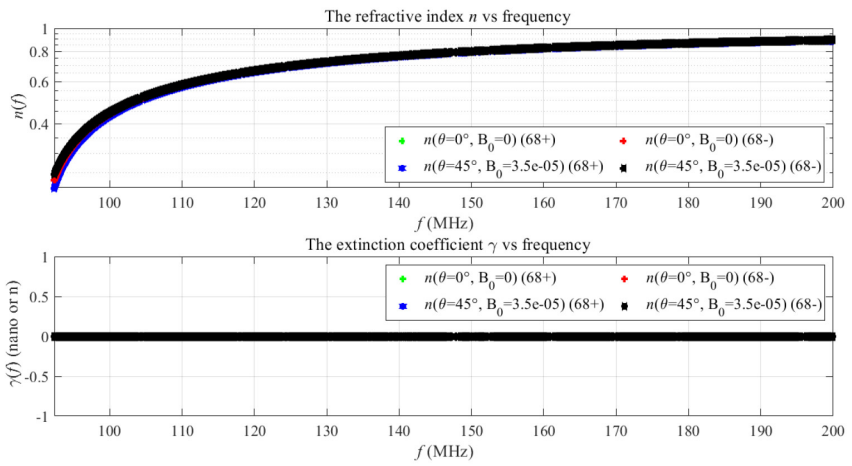

(a) (top) the refractive index, $n$, vs. frequency; (bottom) the extinction coefficient, $\gamma$, vs frequency utilizing (68).
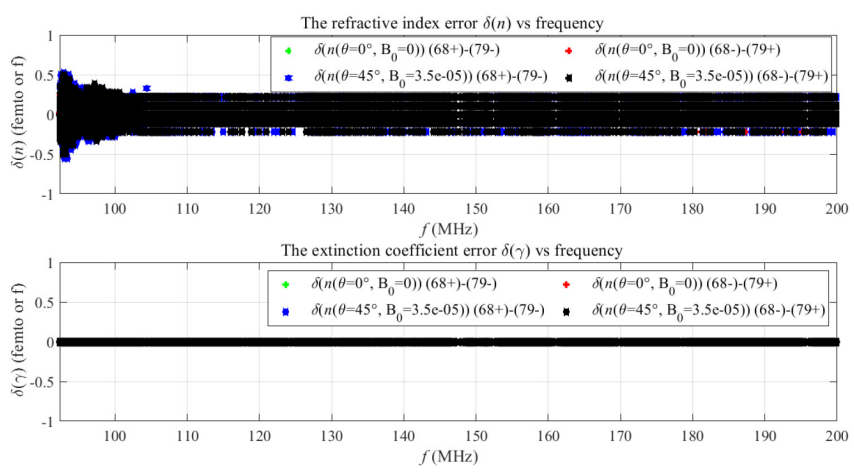

(b) (top) the refractive index error, $\delta(n)$, vs. frequency; (bottom) the extinction coefficient error, $\delta(\gamma)$, vs frequency utilizing (68) and (79).

FIGURE 1: Numerical calculations of the refractive index, extinction coefficient, refractive index error, and extinction coefficient error vs. frequency.
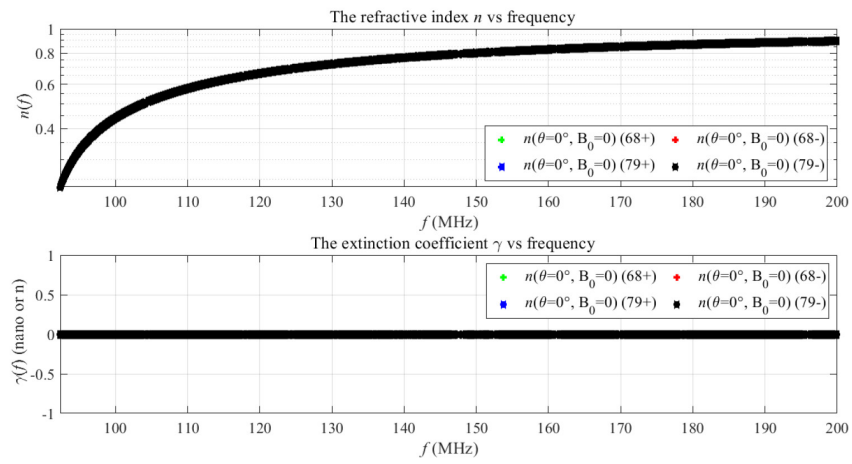

(a) (top) the refractive index, $n$, vs. frequency; (bottom) the extinction coefficient, $\gamma$, vs frequency utilizing (68).

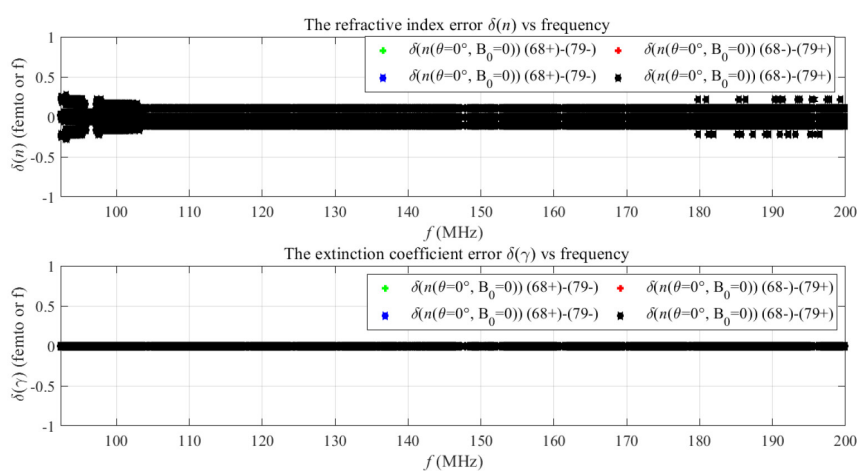

(b) (top) the refractive index error, $\delta(n)$, vs. frequency; (bottom) the extinction coefficient error, $\delta(\gamma)$, vs frequency utilizing (68) and (79).

FIGURE 2: Numerical calculations of the refractive index, extinction coefficient, refractive index error, and extinction coefficient error vs. frequency.
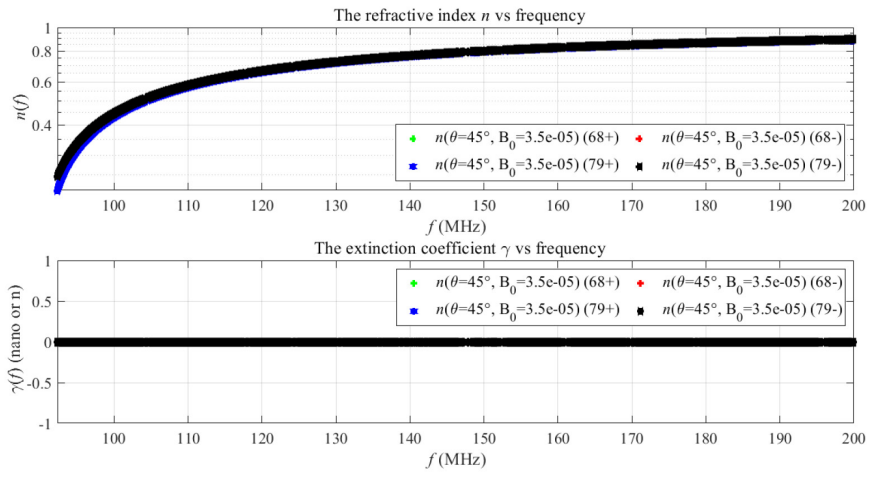

(a) (top) the refractive index, $n$, vs. frequency; (bottom) the extinction coefficient, $\gamma$, vs frequency utilizing (68). 
The terrestrial magnetic field in the lower ionosphere at equatorial latitudes is approximately $B_{0}=0.35 \times 10^{-4}(T)$ [35]. Figure 1 shows the plots of (top) the refractive index, extinction coefficient (68)-(79); (bottom) refractive index error, and extinction coefficient error (68)-(79) vs. frequency. The numerical computations of the refractive index and the extinction coefficient error are zero.

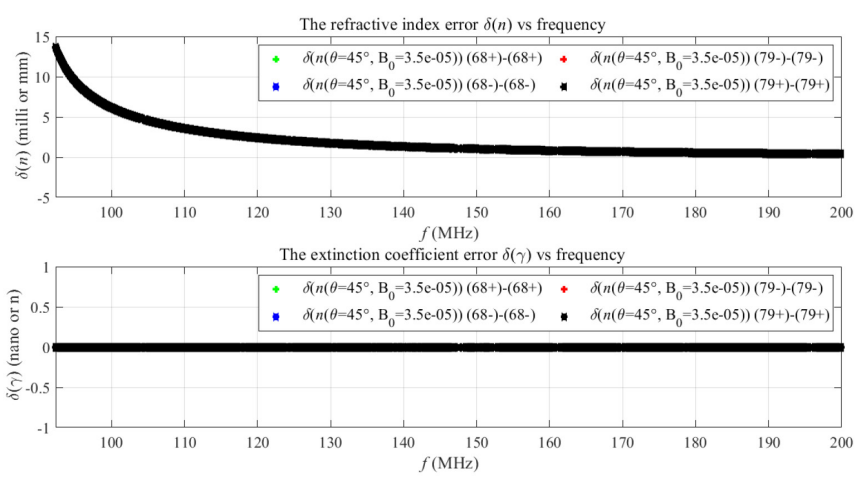

(b) (top) the refractive index error, $\delta(n)$, vs. frequency; (bottom) the extinction coefficient error, $\delta(\gamma)$, vs frequency utilizing (68) and (79).

FIGURE 3: Numerical calculations of the refractive index, extinction coefficient, refractive index error, and extinction coefficient error vs. frequency.
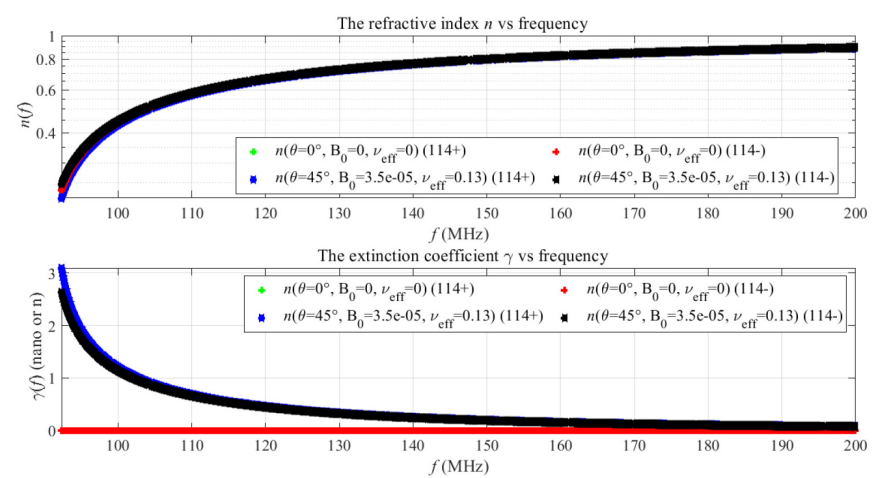

(a) (top) the refractive index, $n$, vs. frequency; (bottom) the extinction coefficient, $\gamma$, vs frequency utilizing (114).
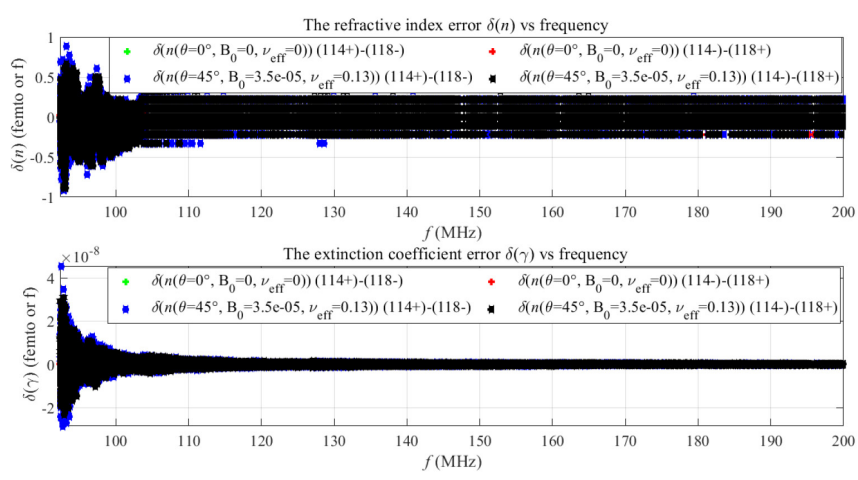

(b) (top) the refractive index error, $\delta(n)$, vs. frequency; (bottom) the extinction coefficient error, $\delta(\gamma)$, vs frequency utilizing (114)/(118).

FIGURE 4: Numerical calculations of the refractive index, extinction coefficient, refractive index error, and extinction coefficient error vs. frequency.

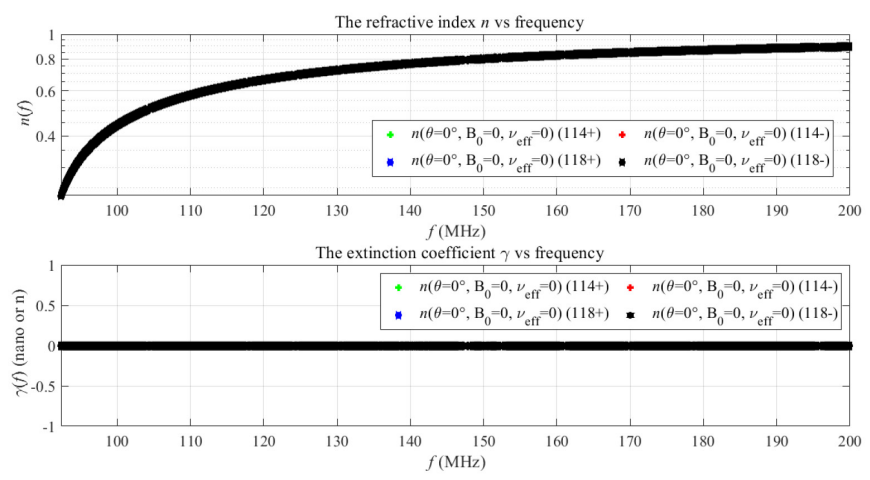

(a) (top) the refractive index, $n$, vs. frequency; (bottom) the extinction coefficient, $\gamma$, vs frequency utilizing $(114) /(118)$.

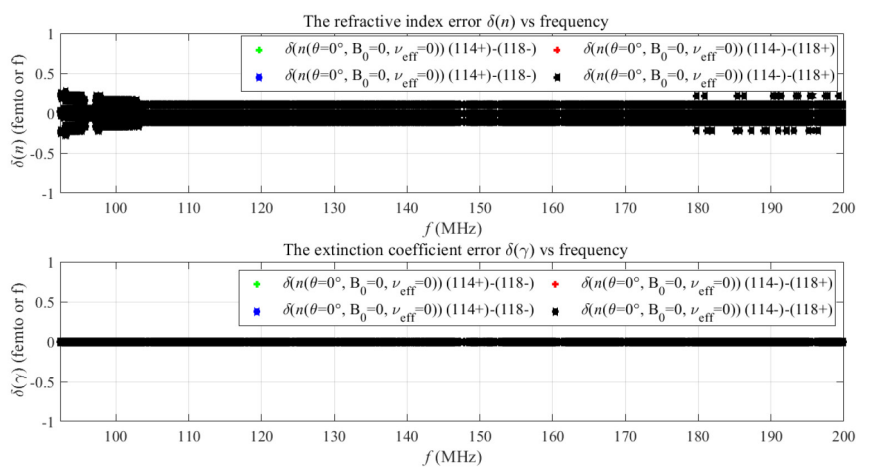

(b) (top) the refractive index error, $\delta(n)$, vs. frequency; (bottom) the extinction coefficient error, $\delta(\gamma)$, vs frequency utilizing (114)/(118).

FIGURE 5: Numerical calculations of the refractive index, extinction coefficient, refractive index error, and extinction coefficient error vs. frequency.

Figure 2 displays exactly the same scenario as in Fig. 1 but for magnetic flux index equal to zero.

Figure 3 contains exactly the same scenario as the data shown in Fig. 1 but for magnetic flux index equal to $0.35 \times 10^{-4} \equiv 3.5 \times 10^{-5}(\mathrm{~T})$ and the elevation angle equal to $45^{\circ}$. 


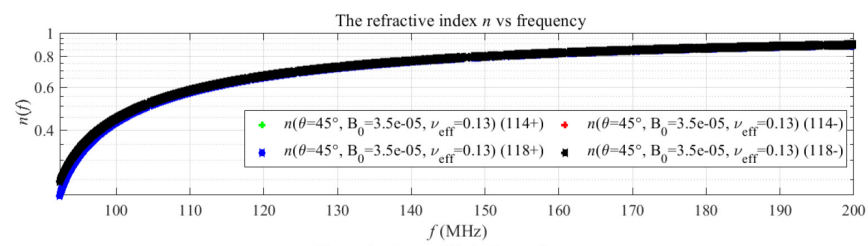

The extinction coefficient $\gamma$ vs frequency

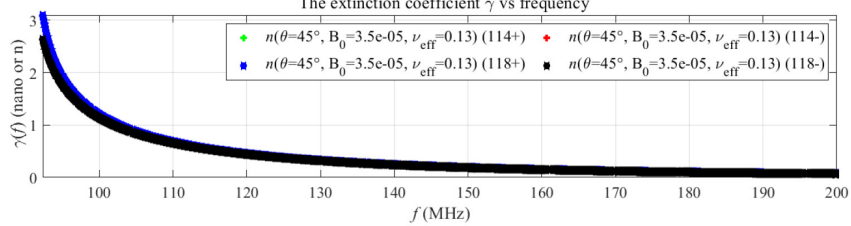

(a) (top) the refractive index, $n$, vs. frequency; (bottom) the extinction coefficient, $\gamma$, vs frequency utilizing (114)/(118).
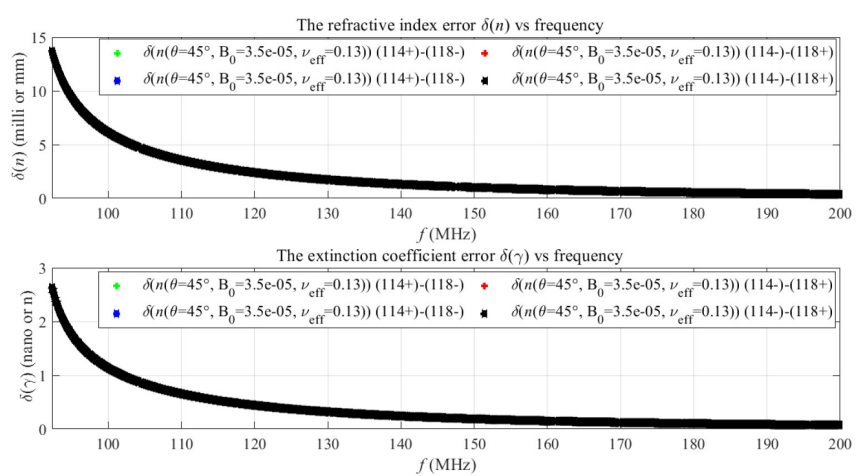

(b) (top) the refractive index error, $\delta(n)$, vs. frequency; (bottom) the extinction coefficient error, $\delta(\gamma)$, vs frequency utilizing (114)/(118).

FIGURE 6: Numerical calculations of the refractive index, extinction coefficient, refractive index error, and extinction coefficient error vs. frequency.

Figure 4 shows the plots of (top) the refractive index, extinction coefficient (114)-(118); (bottom) refractive index error, and extinction coefficient error (114)-(118) vs. frequency. The numerical computations of the refractive index and the extinction coefficient given (114)-(118) shows that the error is zero or bound on the order of $10^{-15}$ for the refractive index and $10^{-23}$ for the extinction coefficient.

\subsection{Refractive Index in Anisotropic Absorptive Turbulent Magnetized Plasma}

In this subsection we present, numerical results of refractive index and of the extinction coefficient using the (114)-(118) (i.e., the collision frequency is assumed to be non-zero).

Numerical results show that we can obtained identical computational, numerical values of the refractive index and of the extinction coefficient given (68)-(79).

Figure 5 depicts exactly the same data as those shown in Fig.
4 but for zero elevation angle and zero external magnetic flux index. As shown in Fig. 5 the refractive index error computed using $(114) /(118)$ is on the order of less than $4 \times 10^{-16}$ and the extinction coefficient error is absolutely zero.

Figure 6 displays exactly the same data as those shown in Fig. 4 but for $45^{\circ}$ elevation angle and $3.5 \times 10^{-5}(\mathrm{~T})$ external magnetic flux index. As shown in Fig. 6 the refractive index error computed using (114)/(118) is on the order of less than 0.15 milli for very low frequencies and decreases as a function of frequency and the extinction coefficient error is less than of 0.3 nano of the same nature as the refractive index error.

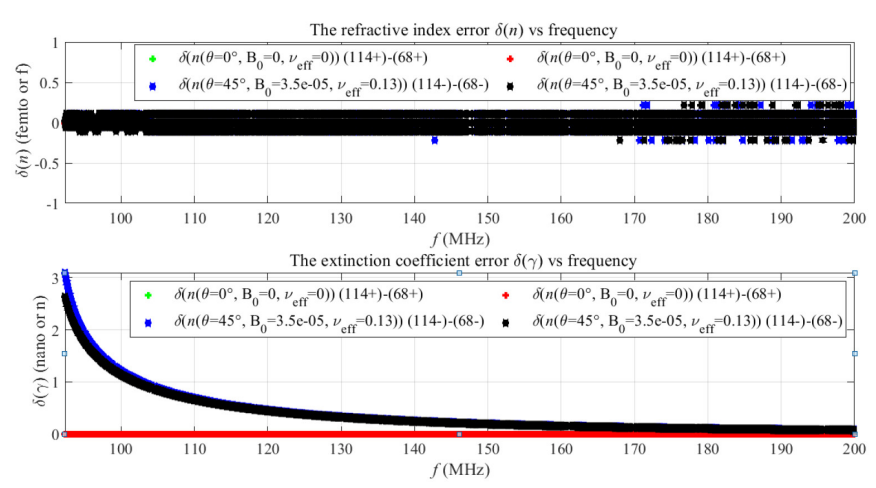

(a) (top) the refractive index error, $\delta(n)$, vs. frequency; (bottom) the extinction coefficient error, $\delta(\gamma)$, vs frequency utilizing (68)/(114).

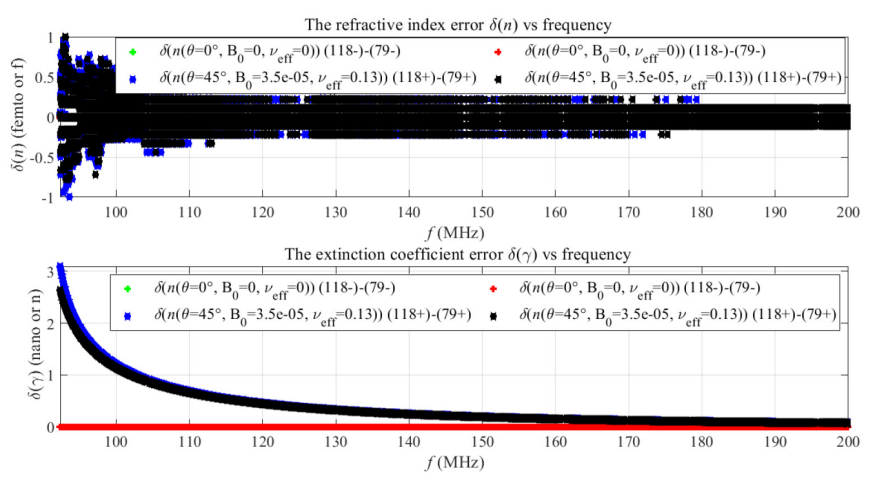

(b) (top) the refractive index error, $\delta(n)$, vs. frequency; (bottom) the extinction coefficient error, $\delta(\gamma)$, vs frequency utilizing $(79) /(118)$.

FIGURE 7: Numerical calculations of the refractive index error and the extinction coefficient error vs. frequency.

Figure 7 displays exactly the same data as those shown in Figs. 1-6 by assessing the effect of the collision frequency using: (a) (114) minus (68) and in (b) (118) minus (79). As shown by the data in Fig. 7.

For zero collision both the $(114) /(118)$ is reduced to $(68) /(79)$. There appears slightly more computation error for frequencies 
lower than $170 \mathrm{MHz}$ coming from using equations (79)/(118) than $(68) /(114)$.

The effect of both elevation and extinction coefficient is significant for low frequencies in the range of less than 200 MHz. The effect of the ISAB (or "fading") in a new profound and meaningful way will be explained in a separate journal paper.

\section{Conclusions}

This journal paper provides a thorough investigation of the refractive index in absorptive turbulent magnetized plasma.

By means of this masterpiece journal paper I have accomplished three main objectives:

First, I believe that Appleton was right when he stated that "The somewhat complicated [refractive index] formula (35) [1932, [2]] may be simplified for certain practical cases."

Second, I believe I have extended the work published by Prof. Kong, 1990, [9].

Third, I believe that I have conducted the most thorough investigation of Jandieri et al. work in 2007, [14] and explained his assumptions, his findings, and provided significant new understanding of his work.

\section{Acknowledgement}

This work was supported by Giftet Inc. executive office.

I want to profoundly thank the MathWorks at Natick, Massachusetts for providing a sponsored MATLAB licence to Giftet Inc. as part of the Indoor Geolocation Systems MATLAB Library development that will enable the results of this work to be published in Dr. Progri pioneer publication Indoor Geolocation Systems - Theory and Applications. Vol. I (Not yet available in print) [54].

I would like to profoundly thank my physics mentor and friend Prof. Niko Thomo at the Polytechnic University of Tirana, Tirana, Albania 1990-1995 for his guidance and believe in me. He believed that I was going to become a prolific scholar in the field of electromagnetic theory.

I would like to profoundly thank Prof. Reinhold Ludwig of WPI, Worcester, MA for his tremendous guidance, encouragement, and his belief in me in 1996 that someday with my analytical skills I may make significant contributions to the study of the electromagnetic theory in ionized plasma.

This journal paper is dedicated to four special men in my life: my grandfather, Xhevdet Progri, my dear father, Fiqiri Progri, my father's first cousin Dr. Peter Demir, and the forty-first President of the United States of America, George H.W. Bush.

\section{References}

[1] E.V. Appleton, M.A.F. Barnett, "On some direct evidence for downward atmospheric reflection of electric rays," Proc. R. Soc. Lond. 109: pp. 621-641, 1925, DOI: https://doi.org/10.1098/rspa.1925.0149.

[2] E.V. Appleton, "Wireless studies of the ionosphere," IEEProc. Wireless Sect. Inst., vol. 7, no. 21, pp. 257-265, Sep. 1932, DOI: https://doi.org/10.1049/pws.1932.0027.

[3] S.K. Mitra, "Report on the present state of our knowledge of the ionosphere," Uni. Calcutta, pp. 131-215, 1935.

[4] H.K. Sen, A.A. Wyller, "Generalization of the AppletonHartree magneto-ionic formula," Physical Review Letters, vol. 4, no. 7, pp. 355-, 1960, DOI: https://doi.org/10.1103/PhysRevLett.4.355.

[5] H. K. Sen, A. A. Wyller, "On the generalization of the Appleton-Hartree magnetoionic formulas," J. Geophys. Res., vol. 65, pp. 3931-3950, Dec. 1960, DOI: https://doi.org/10.1029/JZ065i012p03931.

[6] K. Davies, G.A.M. King "On the validity of some approximations to the Appleton-Hartree formula," J. Res. Nat. Bure. Stand.-D. Radio Prop., vol. 65D, no.4, pp. 323332, July-Aug. 1961.

[7] I.P. Sbkarofsky, "Generalized Appleton-Hartree equation for any degree of ionization and application to the ionosphere," Proc. IRE vol. 49, pp. 1857-1871, 1961, DOI: https://doi.org/10.1109/JRPROC.1961.287714.

[8] A.L. Green, "Early history of the ionosphere," J. Atm. \& Terr. Phys., vol. 36, no. 12, pp 2159-2165, Dec. 1974 ${ }^{\mathrm{xvi}}$, DOI: https://doi.org/10.1016/0021-9169(74)90146-9.

[9] J.A. Kong, Electromagnetic Wave Theory. 2nd ed., New York, NY: John Wiley and Sons, 705 pg., 1990.

[10] S. A. Cummer, "Modeling electromagnetic propagation in the Earth-ionosphere waveguide," IEEE Trans. Ant. \& Prop., vol. 48, no. 9, pp. 1420-1429, 2000.

[11]R.D. Hunsucker, J.K. Hargreaves, The High-Latitude Ionosphere and Its Effects on Radio Propagation. New York, NY: Cambridge University Press, 613 pg., 2003, DOI: https://doi.org/10.1017/CBO9780511535758.002. 
[12]I.F. Progri, “An assessment of indoor geolocation systems," Ph.D. Dissertation, Worcester Polytechnic Institute, May 2003.

[13] B.P. Besser, "Synopsis of the historical development of Schumann resonances," Radio Sci., vol. 42, no. 2, pp. 120, Apr. 2007, DOI: https://doi.org/10.1029/2006RS003495.

[14] G.V. Jandieri, A. Ishimaru, V.G. Jandieri, A.G. Khantadze, Z.M. Diasamidze, "Model computations of angular power spectra for anisotropic absorptive turbulent magnetized plasma," Progr. Electromagn. Res., PIER 70, pp. 307-328, 2007 , http://jpier.org/PIER/pier70/16.07013103.Jandieri.IJKD.p df.

[15]K. Schlegel, "Ionospheric research in Germany prior to Karl Rawer," Adv. Radio Sci., vol. 12, pp. 225-230, Nov. 2014, DOI: https://doi.org/10.5194/ars-12-225-2014.

[16] J. Kinrade, "Ionospheric imaging and scintillation monitoring in the Antarctic and Arctic," Ph.D. Dissertation, University of Bath, 2014.

[17]P.H. Diamond, U. Frisch, Y. Pomeau, "Editorial introduction to the special issue "Plasma physics in the 20th century as told by players"," Eur. Phys. J. H, vol. no. 43, pp. 337-353, Nov. 2018, DOI: https://doi.org/10.1140/epjh/e2018-90061-5.

[18] Anon., "Early history of ionospheric discovery," Space Academy, June 2019, URL: https://spaceacademy.net.au/library/notes/earlyion.htm.

[19] Anon., "Appleton-Hartree equation," Wikipedia, the free encyclopedia, June 2019, https://en.wikipedia.org/wiki/Appleton\%E2\%80\%93Hartr ee_equation.

[20]Anon., "Edward Victor Appleton," Wikipedia, the free encyclopedia,

June

2019 , https://en.wikipedia.org/wiki/Edward_Victor_Appleton.

[21]Anon., "Douglas Hartree," Wikipedia, the free encyclopedia, June 2019, https://en.wikipedia.org/wiki/Douglas_Hartree.

[22] Anon., "Earth-ionosphere waveguide," Wikipedia, the free encyclopedia, June

2019, https://en.wikipedia.org/wiki/Earth\%E2\%80\%93ionosphe re_waveguide.

[23] Anon., "Ionospheric absorption," Wikipedia, the free encyclopedia, June 2019, https://en.wikipedia.org/wiki/Ionospheric_absorption.
[24]W.H. Campbell, M.H. Rees, "A study of auroral coruscations," J. Geophy. Res., Jan. 1961, DOI: https://doi.org/10.1029/JZ066i001p00041.

[25] H.E. Hinteregger, "Absorption spectrometric analysis of the upper atmosphere in the EUV region," J. Atm. Sci., vol. 19, no. 5, pp. 351-368, Sep. 1962.

[26] Anon., United States Space Science Program: Report to COSPAR, National Research Council (US), 549 pg., May 1966.

[27] W.I. Thompson, “Atmospheric transmission handbook: A survey of electromagnetic wave transmission in the Earth's atmosphere over the frequency (Wavelength) Range $3 \mathrm{KHz}$ $(100 \mathrm{~km})-300 \mathrm{THz}(0.1 \mu \mathrm{m})$, , Technical Report, Feb. 1971.

[28]K. Prikner, M. Hvoždara, "Characteristics of the ionospheric correction in ULF wave diagnostics of the magnetosphere," Stud. Geophys. Geod., vol. 26, 201, 1982, DOI: https://doi.org/10.1007/BF01582314.

[29] S.C. Coroniti, "Global thunderstorm activity research survey," Technical Report, Atmospheric Sciences Division, Space Science Laboratory, May 1982.

[30]E.A. Schumer "Improved modeling of midlatitude Dregion ionospheric absorption of high frequency radio signals during solar x-ray flares," Ph.D. Dissertation, Air Force Institute of Technology, Department of Engineering Physics, 234 pg., June 2009, https://apps.dtic.mil/dtic/tr/fulltext/u2/a516063.pdf.

[31] Anon., "Centimetre-gram-second system of units," Wikipedia, the free encyclopedia, May 2019, https://en.wikipedia.org/wiki/Centimetre\%E2\%80\%93gra $\mathrm{m} \% \mathrm{E} 2 \% 80 \% 93$ second_system_of_units.

[32] Anon., "Gaussian units," Wikipedia, the free encyclopedia, May 2019, https://en.wikipedia.org/wiki/Gaussian_units.

[33]Anon., "Plasma_(physics)," Wikipedia, the free encyclopedia, Jan. 2019, https://en.wikipedia.org/wiki/Plasma_(physics).

[34]Anon., "Plasma parameters," Wikipedia, the free encyclopedia, Jan. 2019 , https://en.wikipedia.org/wiki/Plasma_parameters.

[35]Anon., (Thomas A. Mehlhorn Superintendent) "NRL plasma formulary," NRL/PU/6790--18-640, Plasma Physics Division, 74 pg., 2018, https://nrl.navy.mil/ppd/sites/nrl.navy.mil.ppd/files/pdfs/ NRL_FORMULARY_18.pdf. 
[36] K.M. Aggarwal, Nardsder Nath, C.S.G.K. Setty, "Collision frequency and transport properties of electrons in the ionosphere," Planet. Space Sci., vol. 27, no. 6, pp. 753-768, 1979.

[37] M. Nishino, S. Nozawa, J.A. Holtet, "Daytime ionospheric absorption features in the polar cap associated with poleward drifting F-region plasma patches," Earth, Planets \& Space, vol. 50, no. 2, pp. 107-117, 1998.

[38]Anon., "Plasma_(oscillation)," Wikipedia, the free encyclopedia, Jan. 2019 , http://en.wikipedia.org/wiki/Plasma_oscillation.

[39] Anon., "Gyroradius," Wikipedia, the free encyclopedia, Dec. 2018, http://en.wikipedia.org/wiki/Gyroradius.

[40] Anon., "Permittivity," Wikipedia, the free encyclopedia, Apr. 2019, https://en.wikipedia.org/wiki/Permittivity.

[41]Anon., "Refractive index," Wikipedia, the free encyclopedia, Dec. 2018, https://en.wikipedia.org/wiki/Refractive_index.

[42] Anon., "Einstein notation," Wikipedia, the free encyclopedia, Jan. 2019, https://en.wikipedia.org/wiki/Einstein_notation.

[43] Anon., "Gradient," Wikipedia, the free encyclopedia, Jan. 2019, https://en.wikipedia.org/wiki/Gradient.

[44]Anon., "Tensor product," Wikipedia, the free encyclopedia, Jan. 2019, https://en.wikipedia.org/wiki/Tensor_product.

[45] Anon., "Dyadics," Wikipedia, the free encyclopedia, Jan. 2019, https://en.wikipedia.org/wiki/Dyadics

[46] Anon., "Metric tensor," Wikipedia, the free encyclopedia, Jan. 2019, https://en.wikipedia.org/wiki/Metric_tensor.

[47]Anon., "Levi-Civita connection," Wikipedia, the free encyclopedia, Jan. 2019, https://en.wikipedia.org/wiki/Levi-Civita_connection.

[48] P.M. Kintner, Jr., “A beginner's guide to space weather and GPS," Private Commun., 11 pp., 2006, http://gps.ece.cornell.edu/SpaceWeatherIntro_ed2_10-3106 ed.pdf.

[49] S. Zhang, S. Lim, C. Rizos, J. Guo, "Atmosphere decomposition for VRS based network-RTK system," in Proc. $22^{\text {nd }}$ Inter. Tech. Mtg. Sat. Div. ION (ION GNSS 2009), Savannah, GA, pp. 2707-2716, Sep. 2009.

[50] Anon., "Determinant," Wikipedia, the free encyclopedia, Dec. 2017, http://en.wikipedia.org/wiki/Determinant.
[51]Anon., "L'Hôpital's rule," Wikipedia, the free encyclopedia, Oct. 2018 , http://en.wikipedia.org/wiki/L'H\%C3\%B4pital's_rule.

[52] G.B. Arfken, H.J., Weber, Mathematical Methods for Physicists, San Diego, CA: Academic Press, 1995.

[53]Anon., "Laplace operator," Wikipedia, the free encyclopedia, Dec. 2013, http://en.wikipedia.org/wiki/Laplace_operator.

[54]I. Progri, Indoor Geolocation Systems-Theory and Applications. Vol. I, $1^{\text {st }}$ ed., Worcester, MA: Giftet Inc., $\sim 800$ pp., 2020 (not yet available in print).

[55]I. Progri, Geolocation of RF Signals-Principles and Simulations. $1^{\text {st }}$ ed., New York, NY: Springer SBM, LLC, 330 pp., Jan. 2011, DOI: http://doi.org/10.1007/978-14419-7952-0,

URL: http://springer.com/engineering/electronics/book/97 8-1-4419-7951-3.

[56]Anon, "MATLAB 2018a," The MathWorks, Inc., Natick, MA, Copyright (C) 1994-2018, The MathWorks, Inc., http://mathworks.com/products/new_products/release200 6a.html?requestedDomain=mathworks.com.

\section{Appendix A: The Simplification of Refractive Index Given by (76)}

The simplification of the refractive index given by (76) is very important. Equation (75) is given by

$$
n^{2}=\frac{1}{\varepsilon_{0}} \frac{2}{\frac{1 \varrho\left(1+\cos ^{2} \theta\right)}{\varepsilon_{0}}+\frac{1 \sin ^{2} \theta}{\varepsilon_{0} \quad \vartheta} \pm \sqrt{\frac{\left(\frac{\varrho}{\delta}-\frac{1}{\vartheta}\right)^{2} \sin ^{4} \theta}{\varepsilon_{0}^{2}}+\frac{4 \cos ^{2} \theta}{\varepsilon_{0}^{2}}\left(\frac{v \sqrt{u}}{\delta}\right)^{2}}}
$$

Where

$$
\begin{aligned}
& \varrho=\vartheta-u \\
& \delta=\vartheta^{2}-u
\end{aligned}
$$

Which can be further written as

$$
n^{2}=\frac{2}{\frac{\varrho \vartheta\left(1+\cos ^{2} \theta\right)+\delta \sin ^{2} \theta \pm \sqrt{(\varrho \vartheta-\delta)^{2} \sin ^{4} \theta+4 v^{2} \vartheta^{2} u \cos ^{2} \theta}}{\delta \vartheta}}
$$

Hence, the elimination of $\varepsilon_{0}$ in Jandieri et al. 2007, [14].

Let us see if we can perform any simplifications to the above equation. Consider the term in front of $\sin ^{4} \theta$ and define it as

$(\vartheta-u) \vartheta-\left(\vartheta^{2}-u\right)=\vartheta^{2}-\vartheta u-\vartheta^{2}+u$

Which is equivalent with

$u-\vartheta u=u-u+u v \equiv u v$

Next, we simplify the following expression 


$$
\begin{aligned}
& \varrho \vartheta\left(1+\cos ^{2} \theta\right)+\delta \sin ^{2} \theta=\mid \begin{array}{c}
\left(\vartheta^{2}-u \vartheta\right)\left(1+\cos ^{2} \theta\right) \\
+\delta \sin ^{2} \theta
\end{array} \\
&=\mid \begin{array}{c}
\vartheta^{2}-u \vartheta+\vartheta^{2} \cos ^{2} \theta \\
-u \vartheta \cos ^{2} \theta+\vartheta^{2} \sin ^{2} \theta-u \sin ^{2} \theta \\
\end{array}=2 \vartheta^{2}-u \vartheta-u+u v \cos ^{2} \theta \\
&=2 \vartheta^{2}-2 u \vartheta-u v \operatorname{csin}^{2} \theta
\end{aligned}
$$

Next, substituting (124) and (125) into the expression of the refractive index (122) and then perform some straightforward factorization yields

$$
n^{2}=\frac{2\left(\vartheta^{2}-u\right) \vartheta}{2 \vartheta(\vartheta-u)-u v \sin ^{2} \theta \pm v \sqrt{u^{2} \sin ^{4} \theta+4 \vartheta^{2} u \cos ^{2} \theta}}
$$

This concludes the derivations of Appendix A.

\section{Appendix B: The Derivations of Kong (14) pg. 78}

Equation (14) in Kong, 1990 [9] pg. 78 a formula, known as the Appleton-Hartree formula [2], [19], derived from polarizationcurrent arguments.

As I stated in the introduction of the paper, Appleton states "The somewhat complicated [refractive index] formula (35) [1932, [2]] may be simplified for certain practical cases". But I could not find a publication that contained all these simplifications. Therefore, I contacted the Prof. Kong publisher and I was offered help to perform the complete derivations of the second to the third line of (14) Kong, 1990 [9] pg. 78.

$$
n^{2}=1-v \frac{2 \vartheta^{2}-u \sin ^{2} \theta \pm \sqrt{u^{2} \sin ^{4} \theta+4 \vartheta^{2} u \cos ^{2} \theta}}{2\left(\vartheta^{2}-u\right)+u v\left(1+\cos ^{2} \theta\right) \pm v \sqrt{u^{2} \sin ^{4} \theta+4 \vartheta^{2} u \cos ^{2} \theta}}
$$

For simplicity let us define

$$
\begin{aligned}
& x=2 \vartheta^{2}-u \sin ^{2} \theta \\
& y=u^{2} \sin ^{4} \theta+4 \vartheta^{2} u \cos ^{2} \theta \\
& z=2\left(\vartheta^{2}-u\right)+u v\left(1+\cos ^{2} \theta\right)
\end{aligned}
$$

Then substituting (128), (129), and (130) into (127) we have

$$
1-n^{2}=v \frac{x \pm \sqrt{y}}{\mathrm{z} \pm v \sqrt{y}}
$$

Next, we multiply both sides of the numerator and denominator with $x \mp \sqrt{y}$ then (131) is further reduced to

$$
1-n^{2}=v \frac{(x \pm \sqrt{y})(x \mp \sqrt{y})}{(z \pm v \sqrt{y})(x \mp \sqrt{y})}=v \frac{x^{2}-y}{(z x-v y) \mp(z-v x) \sqrt{y}}
$$

Next, let us perform the computations of the terms in (132)

$$
\begin{aligned}
x^{2}-y & =\left(2 \vartheta^{2}-u \sin ^{2} \theta\right)^{2}-u^{2} \sin ^{4} \theta-4 \vartheta^{2} u \cos ^{2} \theta \\
& =4 \vartheta^{4}-4 \vartheta^{2} u \sin ^{2} \theta-4 \vartheta^{2} u \cos ^{2} \theta \\
& =4 \vartheta^{2}\left(\vartheta^{2}-u\right) \\
z x-v y & =\mid \begin{array}{c}
\left(2 \vartheta^{2}-2 u+u v\left(1+\cos ^{2} \theta\right)\right)\left(2 \vartheta^{2}-u \sin ^{2} \theta\right) \\
-u^{2} v \sin ^{4} \theta-4 \vartheta^{2} v u \cos ^{2} \theta
\end{array} \\
& =2 \vartheta\left(\vartheta^{2}-u\right)\left(2 \vartheta-u \sin ^{2} \theta\right) \\
z-v x= & 2 \vartheta^{2}-2 u+u v\left(1+\cos ^{2} \theta\right)-2 \vartheta^{2} v+u v \sin ^{2} \theta \\
& =2 \vartheta^{3}-2 u \vartheta=2 \vartheta\left(\vartheta^{2}-u\right)
\end{aligned}
$$

Substituting (133), (134), and (135) into (132) yields

$$
1-n^{2}=v \frac{2 \vartheta}{2 \vartheta-u \sin ^{2} \theta \mp \sqrt{u^{2} \sin ^{4} \theta+4 \vartheta^{2} u \cos ^{2} \theta}}
$$

This concludes the derivations of Appendix B.

\section{Appendix C: A Brief Discussion on Electron Collision Frequency}

The electron collision frequency in a completely ionized ionosphere is given by [4], [5], [7], [34], [35]

$$
\omega_{\text {eff }}=2 \pi \times 2.91 \times 10^{-6} n_{e} \ln \Lambda T_{e}^{-3 / 2}(\mathrm{rad} / \mathrm{s})
$$

where

$n_{e}$ is the plasma electron density.

$\lambda=\ln \Lambda$ is the Coulomb Logarithm [35]. Typically, $\lambda \approx 10-20$

Representative daytime and nighttime ionospheric electron and ion number density profiles and collision frequency profiles vs. altitude (from 0 to $200 \mathrm{~km}$ ) are shown in Fig. 2 of (Cummer 2000 pg. 4, [10]).

\section{Appendix D: The Explanation of Differences between (100)-(102) and (104)-(106)}

Equations (100)-(102) derived from Progri is slightly different from (104)-(106) which are from Jandieri et al, 2007 [14] (1). The obvious question is which one are right and why?

The tiebreaker comes from Kong, 1990 [9] Pr. P.2.1.2. pg. 92.

If we assume that there is no magnetic field, $u=0$ and that the force of the electron is on the $x$ direction then the permittivity tensor $\boldsymbol{\varepsilon}$ given by (100)-(102) is reduced to a scalar as follows

$$
\varepsilon=\varepsilon_{o}\left(1-\frac{v}{1+i s}=1-\frac{v}{1+s^{2}}+i \frac{v s}{1+s^{2}}\right)
$$

Substituting (34) and (103) into the above yields 


$$
\varepsilon=\varepsilon_{o}\left(1-\frac{\frac{\omega_{p}^{2}}{\omega^{2}}}{1+\frac{\omega_{\text {eff }}^{2}}{\omega^{2}}}+i \frac{\frac{\omega_{p}^{2} \omega_{\text {eff }}}{\omega^{2} \omega}}{1+\frac{\omega_{\text {eff }}^{2}}{\omega^{2}}}\right)
$$

Which is equivalent with

$$
\varepsilon=\varepsilon_{o}\left[1-\frac{\omega_{p}^{2}}{\omega^{2}+\omega_{\mathrm{eff}}^{2}}+i \frac{\omega_{p}^{2} \omega_{\mathrm{eff}}}{\omega\left(\omega^{2}+\omega_{\mathrm{eff}}^{2}\right)}\right]
$$

Which is identical to the result in Kong, 1990 [9] Pr. P.2.1.2.pg. 92.

If we were to repeat exactly the same exercise using Jandieri et al, 2007 [14] (1) then the result would be

$$
\varepsilon=\varepsilon_{o}\left[1-\frac{\omega_{p}^{2}}{\omega^{2}+\omega_{\mathrm{eff}}^{2}}-i \frac{\omega_{p}^{2} \omega_{\mathrm{eff}}}{\omega\left(\omega^{2}+\omega_{\mathrm{eff}}^{2}\right)}\right]
$$

Equation (100), (101) are probably OK. Finally, it remains to explain the difference from (102) and (106).

$$
1-\left[\frac{v(1+i s-u)}{(1+i s)^{2}-u} \equiv \frac{v(1+i s-u)}{1+2 i s-s^{2}-u} \equiv \frac{v(1+i s-u)}{(1+i s-u)+(i s)^{2}+i s-i s u+i s u}\right]
$$

Which is equivalent with

$$
1-\left[\frac{v(1+i s-u)}{(1+i s)^{2}-u} \equiv \frac{v(1+i s-u)}{(1+i s-u)(1+i s)+i s u}\right]
$$

Ignoring a second order term isu the above can be approximates as

$$
1-\left[\frac{v(1+i s-u)}{(1+i s)^{2}-u} \cong \frac{v(1+i s-u)}{(1+i s-u)(1+i s)}=\frac{v}{1+i s}\right]
$$

Clearly comparing and contrasting (144) with the term with (106) from Jandieri et al, 2007 [14] (1), the difference is a ' ${ }^{\prime}$ which appears to be the only mistake in Jandieri et al, 2007 [14] (1).

\section{Appendix E: Simplification Calculations with Complex Numbers (108) through (110)}

I believe that it is necessary to preserve the simplification calculations with complex numbers of (108) through (110).

First, simplifying (108) produces

$$
\begin{aligned}
\kappa & =\frac{1}{\varepsilon_{0}} \frac{\left[(1+i s)^{2}-u\right]\left[(1+i s)^{2}-u-v(1+i s)\right]}{\left[(1+i s)^{2}-u-v(1+i s)\right]^{2}-v^{2} u} \\
& =\frac{1}{\varepsilon_{0}} \frac{\left[(1+i s)^{2}-u\right]\left[(1+i s)^{2}-u-v(1+i s)\right]}{\left[(1+i s)^{2}-u\right]^{2}-2\left[(1+i s)^{2}-u\right] v(1+i s)+v^{2}(1-i s)^{2}-v^{2} u} \\
& =\frac{1}{\varepsilon_{0}} \frac{\left[(1+i s)^{2}-u\right]\left[(1+i s)^{2}-u-v(1+i s)\right]}{\left[(1+i s)^{2}-u\right]^{2}-2\left[(1+i s)^{2}-u\right] v(1+i s)+v^{2}\left[(1+i s)^{2}-u\right]}
\end{aligned}
$$

$$
=\frac{1}{\varepsilon_{0}} \frac{(1+i s)^{2}-v(1+i s)-u}{(1+i s)^{2}-2 v(1+i s)+v^{2}-u}
$$

Which produces the desired answer in (108).

Second, simplifying (109) produces

$$
\kappa_{g}=\frac{1}{\varepsilon_{0}} \frac{\left[(1+i s)^{2}-u\right] v \sqrt{u}}{\left[(1+i s)^{2}-u-v(1+i s)\right]^{2}-v^{2} u}
$$

$$
=\frac{1}{\varepsilon_{0}} \frac{\left[(1+i s)^{2}-u\right] v \sqrt{u}}{\left[(1+i s)^{2}-u\right]^{2}-2\left[(1+i s)^{2}-u\right] v(1+i s)+v^{2}\left[(1+i s)^{2}-u\right]}
$$

Which produces the desired answer in (109).

Third, simplifying (110) produces

$$
\kappa_{z}=\frac{1}{\varepsilon_{0}} \frac{(1+i s)^{2}-u}{(1+i s)^{2}-u-v(1+i s-u)}
$$

Which produces the desired answer in (110). This concludes the derivations of Appendix E.

\section{Appendix F: Substitution of $\kappa, \kappa_{g}$, and $\kappa_{z}$ given by (111) through (113) in (68)}

Substituting $\kappa, \kappa_{g}$, and $\kappa_{z}$ given by (96) through (98) in (68) yields,

$$
n^{2}=\frac{2}{x \pm \sqrt{y}}
$$

Where

$$
\begin{aligned}
& x=\frac{a\left(1+\cos ^{2} \theta\right)}{d}+\frac{(1+i s)^{2}-u}{b} \sin ^{2} \theta \\
& y=\left[\frac{a}{d}-\frac{(1+i s)^{2}-u}{b}\right]^{2} \sin ^{4} \theta+4\left(\frac{v \sqrt{u}}{d}\right)^{2} \cos ^{2} \theta \\
& a=(1+i s)^{2}-v(1+i s)-u=(1+i s) q-u \\
& b=a+v u \\
& c=a+v \\
& d=[(1+i s)-v]^{2}-u=q^{2}-u \\
& q=(1+i s)-v
\end{aligned}
$$

Let us see if we can make any simplifications! First, let us evaluate the term in front of $\sin ^{4} \theta$.

$$
\frac{a}{d}-\frac{(1+i s)^{2}-u}{b}=\frac{a b-\left[(1+i s)^{2}-u\right] d}{d b}
$$

Substituting $b$ into the numerator of (156) we obtain 


$$
\frac{a}{d}-\frac{(1+i s)^{2}-u}{b}=\frac{a^{2}+a v u-\left[(1+i s)^{2}-u\right] d}{d b}
$$

Substituting $d$ into the one of the coefficients in the numerator of (157) and performing certain manipulations yields

$$
\frac{a}{d}-\frac{(1+i s)^{2}-u}{b}=\frac{a b-\left[(q+v)^{2}-u\right] d}{b d}=\frac{a b+u d-(q+v)^{2} d}{b d}
$$

First, we evaluate

$$
a b+u d=a(a+v u)+\left(q^{2}-u\right) u
$$

Next, we evaluate

$$
(q+v)^{2} d=(q+v)^{2}\left(q^{2}-u\right)
$$

Substituting (159) and (160) into (158) yields

$$
\frac{a}{d}-\frac{(1+i s)^{2}-u}{b}=\frac{a^{2}+a v u+\left(q^{2}-u\right)\left[u-(q+v)^{2}\right]}{b d}
$$

Next we evaluate

$$
a^{2}-(q+v)^{2} q^{2}=[a-(q+v) q][a+(q+v) q]
$$

which is identical to

$$
a^{2}-(q+v)^{2} q^{2}=u^{2}-2(q+v) q u
$$

Substituting (163) into (161) yields

$$
\frac{a}{d}-\frac{(1+i s)^{2}-u}{b}=\frac{(q+v)^{2} u-2(q+v) q u+q^{2} u+a v u}{b d}
$$

which is identical to

$$
\frac{a}{d}-\frac{(1+i s)^{2}-u}{b}=\frac{\left[(q+v)^{2}-2(q+v) q+q^{2}\right] u+a v u}{b d}
$$

which is the same as

$$
\frac{a}{d}-\frac{(1+i s)^{2}-u}{b}=\frac{v^{2} u+a v u}{b d}
$$

Which finally produces

$$
\frac{a}{d}-\frac{(1+i s)^{2}-u}{b}=\frac{[v+(1+i s) q-u] v u}{d b}=\frac{(a+v) v u}{d b}
$$

Second, let us evaluate the term outside of the square root.

$\frac{a\left(1+\cos ^{2} \theta\right)}{d}=\frac{2 a}{d}-\frac{a \sin ^{2} \theta}{d}=\frac{2 a}{d}-\frac{[(q+v) q-u] \sin ^{2} \theta}{d}$

which is equivalent with

$$
\frac{a\left(1+\cos ^{2} \theta\right)}{d}=\frac{2 a}{d}-\frac{(q+v) q \sin ^{2} \theta}{d}+\frac{u \sin ^{2} \theta}{d}
$$

And

$$
\frac{\left[(1+i s)^{2}-u\right] \sin ^{2} \theta}{b}=\frac{\left[(q+v)^{2}-u\right] \sin ^{2} \theta}{b}
$$

Which is equivalent with

$$
\frac{\left[(1+i s)^{2}-u\right] \sin ^{2} \theta}{b}=\frac{(q+v)^{2} \sin ^{2} \theta}{b}-\frac{u \sin ^{2} \theta}{b}
$$

Substituting (158) and (171) into the term outside of the square root yields

$$
\begin{aligned}
& \left|\begin{array}{c|c}
\frac{a\left(1+\cos ^{2} \theta\right)}{d} \\
+\frac{\left[(1+i s)^{2}-u\right] \sin ^{2} \theta}{b}
\end{array}=\right| \begin{array}{l}
\frac{2 a}{d}+\left[\frac{u}{d}-\frac{(q+v) q}{d}\right] \sin ^{2} \theta \\
{\left[+\frac{(q+v)^{2}}{b}-\frac{u}{b}\right] \sin ^{2} \theta}
\end{array} \\
& =\frac{2 a}{d}+\frac{\{(q+v)[(q+v) d-q b]+u(b-d)\} \sin ^{2} \theta}{b d} \\
& =\frac{2 a}{d}-\frac{a(b-d) \sin ^{2} \theta}{b d}+\frac{v d(q+v) \sin ^{2} \theta}{b d}
\end{aligned}
$$

Next we evaluate the term,

$$
\begin{aligned}
b-d & =a+v u-q^{2}+u \\
& =(q+v) q+v u-q^{2} \\
& =q v+v u
\end{aligned}
$$

Next, substituting (173) into (172) yields

$$
\begin{aligned}
\left.\right|_{+\frac{\left[(1+i s)^{2}-u\right] \sin ^{2} \theta}{b}} ^{\frac{a\left(1+\cos ^{2} \theta\right)}{d}} & =\frac{2 a}{d}+\frac{(q d+v d-a q-a u) v \sin ^{2} \theta}{b d} \\
& =\frac{2 a}{d}+\frac{[(d-a) q+v d-a u] v \sin ^{2} \theta}{b d}
\end{aligned}
$$

Next, we evaluate

$$
d-a=q^{2}-(q+v) q=-q v
$$

Next, we evaluate

$$
v d-a u=\left(q^{2}-u\right)(v-u)-q v u
$$

Next, substituting (176) and (175) into (174) we obtain the following

$$
\frac{a\left(1+\cos ^{2} \theta\right)}{d}+\frac{\left[(1+i s)^{2}-u\right] \sin ^{2} \theta}{b}=\frac{2 a}{d}-\frac{\left(q^{2}+q v-u+v\right) u v \sin ^{2} \theta}{b d}
$$

$$
=\frac{2 a}{d}-\frac{u v(v+a)}{b d} \sin ^{2} \theta
$$

Next, substituting (158) and (177) into (148)-(150) we obtain the following

$n^{2}=\frac{2 b d}{2 a b-u v(v+a) \sin ^{2} \theta \pm v \sqrt{(a+v)^{2} u^{2} \sin ^{4} \theta+4 u b^{2} \cos ^{2} \theta}}$

Next, we evaluate

$n^{2}=\frac{2 b d}{2 a b-v u c \sin ^{2} \theta \pm v \sqrt{u^{2} c^{2} \sin ^{4} \theta+4 u b^{2} \cos ^{2} \theta}}$

If we set $s=0$ in (151)-(155) then the following is obtained

$$
\begin{aligned}
& a=1-v-u \\
& b=a+v u
\end{aligned}
$$




$$
\begin{aligned}
c & =a+v \\
d & =(1-v)^{2}-u=q^{2}-u \\
q & =1-v \equiv \vartheta \\
n^{2} & =\frac{2 \delta(\varrho+v u)}{2 \varrho(\varrho+v u)-v u \vartheta \sin ^{2} \theta \pm v \sqrt{u^{2} \vartheta^{2} \sin ^{4} \theta+4 u(\varrho+v u)^{2} \cos ^{2} \theta}} \\
& =\frac{2 \delta(1-u) \vartheta}{2 \varrho(1-u) \vartheta-v u(1-u) \sin ^{2} \theta \pm v(1-u) \sqrt{u^{2} \sin ^{4} \theta+4 u \vartheta^{2} \cos ^{2} \theta}} \\
& =\frac{2\left(\vartheta^{2}-u\right) \vartheta}{2(\vartheta-u) \vartheta-v u \sin ^{2} \theta \pm v \sqrt{u^{2} \sin ^{4} \theta+4 u \vartheta^{2} \cos ^{2} \theta}} \\
& =\frac{2\left[(1-v)^{2}-u\right](1-v)}{2\left[(1-v)^{2}-u\right]+v u+v u \cos ^{2} \theta \pm v \sqrt{u^{2} \sin ^{4} \theta+4 u(1-v)^{2} \cos ^{2} \theta}}
\end{aligned}
$$

Which is identical to (75).

Next, employing the identity (61)

$$
\begin{gathered}
n^{2}=1-\left(1-\frac{2 b d}{2 a b-v u c \sin ^{2} \theta \pm v \sqrt{u^{2} c^{2} \sin ^{4} \theta+4 u b^{2} \cos ^{2} \theta}}\right) \\
=1-\frac{2 a b-v u c \sin ^{2} \theta \pm v \sqrt{u^{2} c^{2} \sin ^{4} \theta+4 u b^{2} \cos ^{2} \theta}-2 b d}{2 a b-v u c \sin ^{2} \theta \pm v \sqrt{u^{2} c^{2} \sin ^{4} \theta+4 u b^{2} \cos ^{2} \theta}}
\end{gathered}
$$

Substituting (175) into (186) we obtain the following

$$
\begin{aligned}
& n^{2}=1-\frac{2(a-d) b-v u c \sin ^{2} \theta \pm v \sqrt{u^{2} c^{2} \sin ^{4} \theta+4 u b^{2} \cos ^{2} \theta}}{2 a b-v u c \sin ^{2} \theta \pm v \sqrt{u^{2} c^{2} \sin ^{4} \theta+4 u b^{2} \cos ^{2} \theta}} \\
= & 1-v \frac{2 q b-u c \sin ^{2} \theta \pm \sqrt{u^{2} c^{2} \sin ^{4} \theta+4 u b^{2} \cos ^{2} \theta}}{2 a b-v u c \sin ^{2} \theta \pm v \sqrt{u^{2} c^{2} \sin ^{4} \theta+4 u b^{2} \cos ^{2} \theta}}
\end{aligned}
$$

This concludes the derivations of Appendix E.

\section{Appendix G: The Simplification of Appleton- Hartree magneto-ionic formula given by Progri (116)}

Similar to (14) in Kong, 1990 [9] pg. 78 a formula, known as the Appleton-Hartree magneto-ionic formula [2], [19], derived from polarization-current arguments, we derive here a simplified formula using complex algebra. From Appendix B, (131) we already know that the simplified equation can be obtained if we initially perform a series of simplifications.

First, from (187) let us define $x, y$, and $z$

$x=2 q b-u c \sin ^{2} \theta$

$y=u^{2} c^{2} \sin ^{4} \theta+4 u b^{2} \cos ^{2} \theta$

$$
z=2 a b-v u c \sin ^{2} \theta
$$

Then substituting (188)-(190) into (187) we obtain (131). If, we multiply both sides of the numerator and denominator of (131) with $x \mp \sqrt{y}$ then (131) is further reduced to (132).

Second, we evaluate $x^{2}-y$ as follows

$$
\begin{aligned}
x^{2}-y & =\left(2 q b-u c \sin ^{2} \theta\right)^{2}-\left(u^{2} c^{2} \sin ^{4} \theta+4 u b^{2} \cos ^{2} \theta\right) \\
& =4 b^{2} q^{2}-4 b q u c \sin ^{2} \theta-4 u b^{2} \cos ^{2} \theta \\
& =4 b^{2}\left(q^{2}-u\right)-4 b u(q c-b) \sin ^{2} \theta
\end{aligned}
$$

Next, we evaluate

$$
\begin{aligned}
q c-b & =q(a+v)-(a+v u) \\
& =q\left(q^{2}-u+q v+v\right)-\left(q^{2}-u+q v+v u\right) \\
& =\left(q^{2}-u\right)(q+v-1)
\end{aligned}
$$

Next, substituting (192) into (191) we obtain

$$
\begin{aligned}
x^{2}-y & =4 b\left(q^{2}-u\right)\left[b-u(q+v-1) \sin ^{2} \theta\right] \\
& =4 b\left(q^{2}-u\right)\left[b-i \operatorname{susin}^{2} \theta\right]
\end{aligned}
$$

Next, let us compute $z x-v y$

$$
\begin{aligned}
z x-v y & =\mid \begin{array}{c}
\left(2 q b-u c \sin ^{2} \theta\right)\left(2 a b-v u c \sin ^{2} \theta\right) \\
-v u^{2} c^{2} \sin ^{4} \theta-v 4 u b^{2} \cos ^{2} \theta
\end{array} \\
& =\left.2 b\right|_{\begin{array}{c}
2 q a b-q v u c \sin ^{2} \theta \\
-a u c \sin ^{2} \theta-2 v u b\left(1-\sin ^{2} \theta\right)
\end{array}} \\
& =\left.2 b\right|_{+u(2 v b-q v c-a c) \sin ^{2} \theta} ^{2 b(q a-v u)}
\end{aligned}
$$

Next, let us compute $q a-v u$

$$
\begin{aligned}
q a-v u & =\left(q^{2}-u+q v\right) q-v u \\
& =(q+v)\left(q^{2}-u\right)
\end{aligned}
$$

Next, let us compute $2 v b-q v c-a c$

$$
\begin{aligned}
2 v b-q v c-a c & =\mid \begin{array}{c}
2 v\left(q^{2}-u+q v+v u\right) \\
-\left(q^{2}-u+2 q v\right)\left(q^{2}-u+q v+v\right)
\end{array} \\
& =\mid \begin{array}{c}
\left(q^{2}-u+q v\right)\left[v-\left(q^{2}-u+2 q v\right)\right] \\
+2 v^{2} u-q v^{2}
\end{array} \\
= & \left(q^{2}-u\right)[v+u-(q+v)(q+2 v)](196)
\end{aligned}
$$

Finally, substituting (195) and (196) into (194) we obtain

$$
z x-v y=\left.2 b\left(q^{2}-u\right)\right|_{+u \mid} \begin{gathered}
2 b(q+v) \\
v+u \\
-(q+v)(q+2 v)
\end{gathered} \sin ^{2} \theta
$$

Next, let us compute $z-v x$

$$
\begin{aligned}
z-v x & =2 a b-v u c \sin ^{2} \theta-v\left(2 q b-u c \sin ^{2} \theta\right) \\
& =2 a b-2 q b v \\
& =2 b(a-q v) \\
& =2 b\left(q^{2}-u\right)
\end{aligned}
$$

Next, substituting (193), (197), and (198) into (132) we 
obtain

$$
\begin{aligned}
& 1-n^{2}=v \frac{4 b\left(q^{2}-u\right)\left[b-i s u \sin ^{2} \theta\right]}{\left[\begin{array}{c}
2 b\left(q^{2}-u\right)\left\{2 b(q+v)+u[u+v-(q+v)(q+2 v)] \sin ^{2} \theta\right\} \\
\mp 2 b\left(q^{2}-u\right) \sqrt{u^{2} c^{2} \sin ^{4} \theta+4 u b^{2} \cos ^{2} \theta}
\end{array}\right.} \\
& =v \frac{2\left(b-i s u \sin ^{2} \theta\right)}{2 f+u g \sin ^{2} \theta \mp \sqrt{u^{2} c^{2} \sin ^{4} \theta+4 u b^{2} \cos ^{2} \theta}}
\end{aligned}
$$

Where

$$
\begin{aligned}
& f=b(q+v) \\
& g=u+v-(q+v)(q+2 v)
\end{aligned}
$$

This concludes the derivations of Appendix $F$ or the simplification of Appleton-Hartree magneto-ionic formula [2], [19] as given by Progri (102). I believe that I have been able to accomplish what Appleton had in mind that "The somewhat complicated [refractive index] formula (35) [1932, [2]] may be simplified for certain practical cases."

\section{Appendix H: Special Cases}

Special cases play an important part in the calculations of the refractive index.

First of all, what are the acceptable values of $v$ and $u$ ? Since for all theoretical and practical purposes $0 \leq v \leq 1$ and $0 \leq$ $u \leq u_{\mathrm{m}} \ll 1 . v=0$ corresponds to absence of plasma; i.e., free-space or $n^{2}=1$ and $v=1$ maximum plasma or $n^{2}=0$. Hence,

$$
\begin{aligned}
& n^{2}(v=0)=\frac{2(1-u) \times 1}{2(1-u)}=1 \\
& n^{2}(v=1)=\frac{0}{-u+u \cos ^{2} \theta \pm u \sin ^{2} \theta}=\mid \begin{array}{cc}
\frac{0}{0} & (+) \\
0 & (-) \cap \theta \neq 0^{\circ}
\end{array}
\end{aligned}
$$

In order to remove the uncertainty in case of $(+)$ we should use the L'Hôpital's rule [51] as follows:

$$
\begin{gathered}
n_{(+)(v=1)}^{2}=\lim _{v \rightarrow 1} \frac{-6 \vartheta^{2}+2 u}{-\begin{array}{c}
-4 \vartheta+u\left(1+\cos ^{2} \theta\right)+\sqrt{u^{2} \sin ^{4} \theta+4 \vartheta^{2} u \cos ^{2} \theta} \\
+v \frac{-8 \vartheta u \cos ^{2} \theta}{\sqrt{u^{2} \sin ^{4} \theta+4 \vartheta^{2} u \cos ^{2} \theta}}
\end{array}} \\
=\frac{2 u}{u\left(1+\cos ^{2} \theta\right)+u \sin ^{2} \theta}=1
\end{gathered}
$$

If $\theta=0^{\circ}$ and $u \neq 0$ then

$$
n^{2}\left(\theta=0^{\circ}\right)=\mid \begin{array}{cc}
\frac{1-u}{1-u}=1 & v=0 \\
\frac{-u}{-u \pm \sqrt{u}}=\frac{\sqrt{u}}{\sqrt{u} \mp 1} \approx \sqrt{u} & v=1
\end{array}
$$

If $\theta=0^{\circ}$ and $u=0$ then

$$
n^{2}\left(\theta=0^{\circ}\right)=\frac{\vartheta^{2}}{\vartheta}=\vartheta \equiv 1-v=\mid \begin{array}{ll}
1 & v=0 \\
0 & v=1
\end{array}
$$

If $\theta=90^{\circ}$ and $u \neq 0$ then

$$
n^{2}=\frac{2\left[\vartheta^{2}-u\right] \vartheta}{2\left(\vartheta^{2}-u\right)+u v \pm u v}=\mid \begin{array}{cc}
\frac{\vartheta^{2}-u}{\vartheta-u} & (+) \\
\vartheta & (-)
\end{array}
$$

Furthermore, if $\theta=90^{\circ}$ and $u \neq 0$ and $(+)$ then we have

$$
n^{2}=\frac{\vartheta^{2}-u}{\vartheta-u}=\mid \begin{array}{ll}
1 & v=0 \\
1 & v=1
\end{array}
$$

Behaves as free-space or the absence of plasma.

If $\theta=90^{\circ}$ and $u=0$ then

$$
n^{2}(u=0)=\frac{2\left(\vartheta^{2}-u\right) \vartheta}{2\left(\vartheta^{2}-u\right)+u v \pm u v}=1-v
$$

Next, if $u=u_{\mathrm{m}} \ll 1$ means maximum effect of the magnetic field but still very very small. Hence,

$$
\begin{gathered}
n^{2} u=u_{\mathrm{m}} \\
u_{\mathrm{m}} \ll 1
\end{gathered}=\frac{2\left(\vartheta^{2}-u_{\mathrm{m}}\right) \vartheta}{\mid \begin{array}{l}
2\left(\vartheta^{2}-u_{\mathrm{m}}\right)+u_{\mathrm{m}} v\left(1+\cos ^{2} \theta\right) \\
\pm v \sqrt{u_{\mathrm{m}}^{2} \sin ^{4} \theta+4 \vartheta^{2} u_{\mathrm{m}} \cos ^{2} \theta}
\end{array}}
$$

Clearly, from (78) if $v=0$ then $n=1$. In the absence of DC magnetic field, $u=0$ then $n^{2}=1-v$.

Since for all theoretical and practical purposes $0 \leq v \leq 1$ and $0 \leq u \leq u_{\mathrm{m}} \ll 1 . v=0$ corresponds to the absence of plasma; i.e., free-space or $n^{2}=1$ and $v=1$ maximum plasma or $n^{2}=0$. Hence,

$$
\begin{aligned}
& n^{2}(v=0)=\frac{2(1-u) \times 1}{2(1) \times(1-u)-0}=1 \\
& n^{2}(v=1)=\frac{2(0-u) \times(0)}{2(0)(0-u)-u \sin ^{2} \theta \pm \sqrt{u^{2} \sin ^{4} \theta+0}}=\mid \begin{array}{l}
\frac{0}{0}(+) \\
0(-)
\end{array}
\end{aligned}
$$

where $(+)$ is the case of ordinary waves and $(-)$ of extraordinary waves.

In order to remove the uncertainty, we should use the L'Hôpital's rule [51] as follows, if $\theta \neq 0$ :

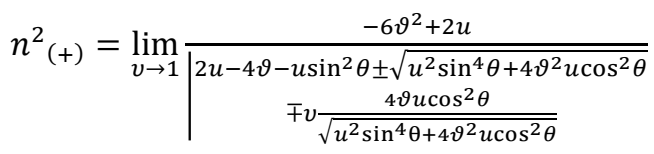

$$
\begin{aligned}
& =\lim _{v \rightarrow 1} \frac{+2 u}{+2 u-u \sin ^{2} \theta \pm \sqrt{\mathrm{u}^{2} \sin ^{4} \theta} \mp 0}=1
\end{aligned}
$$

If $\theta=0^{\circ}$ then

$$
n^{2}{ }_{(+)}=\lim _{v \rightarrow 1} \frac{-6 \vartheta^{2}+2 u}{2 u-4 \vartheta \pm 2 \vartheta \sqrt{u} \mp v 2 \sqrt{u}}
$$

$$
=\lim _{v \rightarrow 1} \frac{2 u}{2 u \mp 2 \sqrt{u}}=\frac{\sqrt{u}}{\sqrt{u} \mp 1} \approx \sqrt{u} ;\left(u_{\mathrm{m}} \ll 1\right)
$$


If $\theta=90^{\circ}$ then

$$
\begin{aligned}
& n_{(+)}^{2}(v=1)=\lim _{v \rightarrow 1} \frac{2 u}{2 u}=1 \text { (ordinary waves) } \\
& n_{(-)}^{2}(v=1)=\lim _{v \rightarrow 1} \frac{2 u}{2 u-2 u=0} \text { (extraordinary waves) }
\end{aligned}
$$

In the case of ordinary waves for $\theta=90^{\circ}$ the ionosphere behaves as if there is a free space if $u \neq 0$; if, $u=0$ then we have a $0 / 0$ uncertainty condition. In the case of extraordinary waves for $\theta=90^{\circ}$ the ionosphere behaves as a mirror; i.e., $n=\infty$ everything is reflected if $u \neq 0$; if, $u=0$ then we have a $0 / 0$ uncertainty condition.

Let us consider next the effect of the magnetic field; i.e., $u=$ 0 means no magnetic field; hence,

$$
n^{2}(u=0)=\frac{2 \vartheta^{2} \vartheta}{2 \vartheta \vartheta-0}=\vartheta=\mid \begin{array}{ll}
0 & v=1 \\
\vartheta & v<1
\end{array}
$$

If there is no magnetic field and maximum plasma then $n^{2}=$ 0 ; or it means that ionosphere absorbs the entire energy of the signal.

Next, let us consider when $u=u_{\mathrm{m}} \ll 1$ means maximum effect of the magnetic field but still very very small; therefore, we obtain.

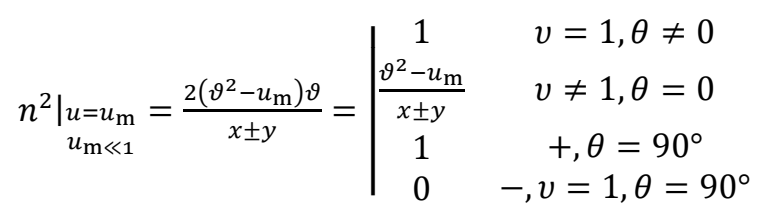

Where

$$
\begin{aligned}
& x=2 \vartheta\left(\vartheta-u_{\mathrm{m}}\right)-u_{\mathrm{m}} v \sin ^{2} \theta \\
& y=v \sqrt{u_{\mathrm{m}}^{2} \sin ^{4} \theta+4 \vartheta^{2} u_{\mathrm{m}} \cos ^{2} \theta}
\end{aligned}
$$

This concludes the derivation of the special cases. Finally we consider the derivation of the extinction coefficient in the absence of magnetic field.

\section{Appendix I: Derivation of the Extinction Coefficient in the Absence of Magnetic Field}

We have already shown in Appendix G that separation of the real and imaginary parts of the Appleton-Hartree magneto-ionic formula is nearly impossible. However, it is possible to perform the separation of the real and imaginary parts in the absence of external magnetic field.

In the absence of DC magnetic field, $u=0$ then

$$
1-n^{2}=\frac{v}{1+i s}
$$

Performing simple complex algebra we obtain

$$
\begin{aligned}
1-n^{2} & =\frac{v(1-i s)}{(1+i s)(1-i s)} \\
& =\frac{v}{1+s^{2}}-\frac{v i s}{1+s^{2}} \\
& =\frac{\frac{\omega_{p}^{2}}{\omega^{2}}}{1+\frac{\omega_{\text {eff }}^{2}}{\omega^{2}}}-\frac{\frac{\omega_{p}^{2}}{\omega^{2}} i s}{1+\frac{\omega_{\text {eff }}^{2}}{\omega^{2}}}
\end{aligned}
$$

Which yields $\Rightarrow$

$$
1-\frac{\frac{\omega_{p}^{2}}{\omega^{2}}}{1+\frac{\omega_{\mathrm{eff}}^{2}}{\omega^{2}}}+i \frac{\frac{\omega_{p}^{2}}{\omega^{2}} \frac{\omega_{\mathrm{eff}}}{\omega}}{1+\frac{\omega_{\mathrm{eff}}^{2}}{\omega^{2}}}=n^{2}
$$

Since, refractive index [40] is a complex quantity; hence, it can be written as

$$
\underline{n}=n+i \gamma
$$

Here, the real part $n$ is the refractive index and indicates the phase velocity, while the imaginary part $\gamma$ is called the extinction coefficient-although $\gamma$ can also refer to the mass attenuation coefficient-and indicates the amount of attenuation when the electromagnetic wave propagates through the material [40].

Taking the square of (224) yields

$$
\underline{n}^{2}=(n+i \gamma)^{2}=n^{2}-\gamma^{2}+i 2 n \gamma
$$

Equating both sides of (225) with (221) yields

$$
1-\underline{n}^{2}=1-n^{2}+\gamma^{2}-i 2 n \gamma=\frac{v}{1+s^{2}}-i \frac{v s}{1+s^{2}}
$$

Or

$$
\begin{aligned}
& 1-n^{2}+\gamma^{2}=\frac{v}{1+s^{2}} \equiv \tilde{v} \\
& 2 n \kappa=\frac{v s}{1+s^{2}} \Rightarrow \gamma=\frac{v s}{2 n\left(1+s^{2}\right)} \Rightarrow \gamma^{2}=\frac{v^{2} s^{2}}{4 n^{2}\left(1+s^{2}\right)^{2}}
\end{aligned}
$$

Substituting (228) into (227) yields

$$
1-n^{2}+\frac{\widetilde{v}^{2} s^{2}}{4 n^{2}}=\tilde{v}
$$

Which is equivalent with

$$
\left(1-n^{2}\right) 4 n^{2}+\tilde{v}^{2} s^{2}=\tilde{v} 4 n^{2}
$$

Which is identical with

$$
4 n^{4}-4(1-\tilde{v}) n^{2}-\widetilde{v}^{2} s^{2}=0
$$

First let us examine

$$
D=4\left[(1-\tilde{v})^{2}+\tilde{v}^{2} s^{2}\right]
$$


From where we find

$$
n^{2}=\frac{2(1-\widetilde{v}) \pm \sqrt{4\left[(1-\widetilde{v})^{2}+\widetilde{v}^{2} s^{2}\right]}}{4}
$$

Since, $n^{2}$ is real and positive; hence, we require that

$$
n^{2}=\frac{1-\widetilde{v}+\sqrt{(1-\widetilde{v})^{2}+\widetilde{v}^{2} s^{2}}}{2}
$$

Which is equivalent with

$$
n^{2}=\frac{1-\frac{v}{1+s^{2}}+\frac{\sqrt{\left(1+s^{2}\right)}}{\left(1+s^{2}\right)} \sqrt{1+s^{2}-2 v+v^{2}}}{2}
$$

\section{Which is identical to}

$$
n^{2}=\frac{1+s^{2}-v+\sqrt{\left(1+s^{2}\right)} \sqrt{(1-v)^{2}+s^{2}}}{2\left(1+s^{2}\right)}
$$

Which is the same as

$$
n^{2}=\frac{1+\sqrt{\frac{(1-v)^{2}+s^{2}}{1+s^{2}}}}{2}-\frac{v}{2\left(1+s^{2}\right)}
$$

The extension coefficient, $\gamma$, can be computed from

$$
\gamma^{2}=\frac{v^{2} s^{2}}{2\left[1+s^{2}-v+\sqrt{\left(1+s^{2}\right)} \sqrt{(1-v)^{2}+s^{2}}\right]\left(1+s^{2}\right)}
$$

This concludes the discussion on Appendix I or derivation of the extinction coefficient in the absence of magnetic field.

medium with high permittivity. Permittivity is the measure of a material's ability to store an electric field in the polarization of the medium. The SI unit for permittivity is farad per meter $\left(\mathrm{F} / \mathrm{m}\right.$ or $\left.\mathrm{F} \mathrm{m}^{-1}\right)$. The lowest possible permittivity is that of a vacuum. Vacuum permittivity, sometimes called the electric constant, is represented by $\varepsilon_{0}$ and has a value of approximately $8.85 \times 10^{-12} \mathrm{~F} / \mathrm{m}$.

In electromagnetism, the electric displacement field $\mathbf{D}$ represents how an electric field $\mathbf{E}$ influences the organization of electric charges in a given medium, including charge migration and electric dipole reorientation. Its relation to permittivity in the very simple case of linear, homogeneous, isotropic materials with "instantaneous" response to changes in electric field is

$$
\mathbf{D}=\varepsilon \mathbf{E}
$$

where the permittivity $\varepsilon$ is a scalar. If the medium is anisotropic, the permittivity is a second rank tensor and it is denoted by $\boldsymbol{\varepsilon}$.

In general, permittivity is not a constant, as it can vary with the position in the medium, the frequency of the field applied, humidity, temperature, and other parameters. In a nonlinear medium, the permittivity can depend on the strength of the electric field. Permittivity as a function of frequency can take on real or complex values.

In SI units, permittivity is measured in farads per meter $(\mathrm{F} / \mathrm{m}$ or $\left.\mathrm{A}^{2} \mathrm{~s}^{4} \mathrm{~kg}^{-1} \mathrm{~m}^{-3}\right)$. The displacement field $\mathbf{D}$ is measured in units of coulombs per square meter $\left(\mathrm{C} / \mathrm{m}^{2}\right)$, while the electric field $\mathbf{E}$ is measured in volts per meter $(\mathrm{V} / \mathrm{m})$. D and $\mathbf{E}$ describe the interaction between charged objects. $\mathbf{D}$ is related to the charge densities associated with this interaction, while $\mathbf{E}$ is related to the forces and potential differences.

v I found another typo in (1) in Jandieri et al, 2007 [14] it was ' $=$ ' it should be '-'.

vi In electromagnetism, absolute impermittivity, often simply called impermittivity, usually denoted by the Greek letter $\kappa$ (kappa), is the measure of the inverse of capacitance that is encountered when forming an electric field in a particular medium. More specifically, impermittivity describes the amount of voltage needed to generate one unit of electric field in a particular medium. Accordingly, a voltage will yield more electric field in a medium with high impermittivity than in a medium with low impermittivity. Impermittivity is the inverse measure of a material's ability to store an electric field in the polarization of the medium. The SI unit for impermittivity is meter per farad $\left(\mathrm{m} / \mathrm{F}\right.$ or $\left.\cdot \mathrm{m} \mathrm{F}^{-1}\right)$. The highest possible impermittivity is that of a vacuum. Vacuum impermittivity, sometimes called the inverse of the electric constant, is represented by $\kappa_{0}$ and has a value of approximately $1.1294 \times 10^{11} \mathrm{~m} / \mathrm{F}$, which is accounts for roughly 75.5 percent of the astronomical unit (AU) distance over 1F. The above definitions of the impermittivity was adopted form the definitions of the permittivity [40].

In electromagnetism, the electric displacement field D represents how an electric field $\mathbf{E}$ influences the organization of electric charges in a given medium, including charge migration and electric dipole reorientation. Its relation to permittivity in the very simple case of linear, homogeneous, isotropic materials with "instantaneous" response to changes in electric field is

$$
\mathbf{E}=\kappa \mathbf{D}
$$
generate one unit of electric flux in a particular medium. Accordingly, a charge will yield more electric flux in a medium with low permittivity than in a

where the impermittivity $\kappa$ is a scalar. If the medium is anisotropic, the 
impermittivity is a second rank tensor and it is denoted by $\boldsymbol{\kappa}$. vii To test that (46) $\boldsymbol{\kappa}$ is in fact the inverse $\boldsymbol{\varepsilon}^{-1}$ we perform the multiplication of (46) with (29) as shown below:

$$
\left[\boldsymbol{\kappa} \equiv \boldsymbol{\varepsilon}^{-1}\right] \cdot \boldsymbol{\varepsilon}=\left[\begin{array}{ccc}
\frac{\varepsilon}{\varepsilon^{2}-\varepsilon_{g}{ }^{2}} & \frac{i \varepsilon_{g}}{\varepsilon^{2}-\varepsilon_{g}{ }^{2}} & 0 \\
\frac{-i \varepsilon_{g}}{\varepsilon^{2}-\varepsilon_{g}{ }^{2}} & \frac{\varepsilon}{\varepsilon^{2}-\varepsilon_{g}{ }^{2}} & 0 \\
0 & 0 & \frac{1}{\varepsilon_{z}}
\end{array}\right] \cdot\left[\begin{array}{ccc}
\varepsilon & -i \varepsilon_{g} & 0 \\
i \varepsilon_{g} & \varepsilon & 0 \\
0 & 0 & \varepsilon_{z}
\end{array}\right]
$$

which yields the identity matrix:

$$
\left[\boldsymbol{\kappa} \equiv \boldsymbol{\varepsilon}^{-1}\right] \cdot \boldsymbol{\varepsilon}=\left[\begin{array}{lll}
1 & 0 & 0 \\
0 & 1 & 0 \\
0 & 0 & 1
\end{array}\right]=\mathbf{I}
$$

thus, completing the proof. viii There is an error in Kong, 1990 [9] top of pg. 78. Equation (67) is the corrected expression of the refractive index squared $n^{2}$.

ix The refractive index, $n$, is always a complex quantity.

$x$ In (68) the ' $+{ }^{\prime}$ sign corresponds to the ordinary waves and ' $-{ }^{\prime}$ sign to the extraordinary waves.

${ }^{x i}$ In (79) the ' $-{ }^{\prime}$ sign corresponds to the ordinary waves and ' $+{ }^{\prime}$ sign to the extraordinary waves. This is very important.

xii One of the main goals of this research is to model absorption of the ionosphere as a function of the electron collision frequency. The study of the electron collision frequency will be subject of a separate journal paper.

xiii Ditto $\mathrm{v}$.

xiv Ditto $x$.

xv Ditto xi.

xvi Originally published in 1946 